\title{
Canadian practice guidelines for surgical intra-abdominal infections
}

\author{
Co-Chairs (listed alphabetically): Anthony W Chow MD FACP FRCPC ${ }^{1}$, Gerald A Evans MD FRCPC ${ }^{2}$, \\ Avery B Nathens MD PhD FRCS MPH ${ }^{3}$ \\ Authors (listed alphabetically): Chad G Ball MSc MD ${ }^{4}$, Glen Hansen PhD ${ }^{5}$, Godfrey KM Harding MD FRCPC ${ }^{6}$, \\ Andrew W Kirkpatrick MD FRCS FACS MHSC ${ }^{4}$, Karl Weiss MD MSc FRCPC ${ }^{7}$, George G Zhanel PhD FCCP6
}

\begin{abstract}
EXECUTIVE SUMMARY
Complicated intra-abdominal infections (IAIs) remain a major challenge in clinical practice. In addition to significant morbidity and mortality for patients, they consume substantial hospital resources. This is compounded by the potential misuse of antimicrobial agents that may result in suboptimal treatment, as well as encourage the selection and spread of antibiotic-resistant microorganisms in the health care setting. The present guideline was developed jointly by the Canadian Surgical Society (CSS) and the Association of Medical Microbiology and Infectious Disease (AMMI) Canada. The primary goal was to provide updated recommendations for the medical and surgical management of complicated IAIs since publication of the 2003 antimicrobial treatment guideline by the Infectious Diseases Society of America (IDSA) (1). Particular focus is directed at risk stratification for poor outcome based on epidemiological studies, current status of antimicrobial susceptibility and resistance profiles among enteric pathogens, therapeutic efficacy of antimicrobial regimens based on randomized clinical trials, operative versus percutaneous approaches for source control, the role of intraabdominal hypertension (IAH) and abdominal compartment syndrome (ACS) in IAI, and infection control and preventive measures for postoperative IAIs and surgical site infections. An additional objective is to categorize the recommendations according to the strength and quality of the available evidence using a standardized grading system. Importantly, the current guideline provides recommendations for initial empirical antimicrobial management of complicated IAIs based on clinical settings and issues unique to the Canadian health care system.

Summarized below are the key evidence-based recommendations grouped according to the main sections discussed in more detail in the guideline. Each recommendation is rated by the strength of support (category A to C) and quality of evidence (grade 1 to 3 ) as assessed by the working group of the guideline.
\end{abstract}

Key recommendations for risk assessment and stratification Recommendation 1. Categorize the severity of illness by using the Acute Physiology and Chronic Health Evaluation II (APACHE II) score: low-moderate (lower than 15$)$ or high (15 or greater) (A-2 evidence). Although the APACHE II scoring is infrequently used clinically outside of the critical care setting at present, it is recommended that physicians and surgeons consider introducing it into clinical use in patients with IAIs. A user-friendly APACHE II calculator can be found on the following Web site $<$ www.globalrph.com/apacheii.htm>.

Recommendation 2. Identify high-risk patients for poor outcome by stratification according to community-acquired versus health care-associated IAIs, previous antibiotic exposure, and underlying comorbid conditions such as diabetes, severe cardiopulmonary disease or immunosuppression (A-2 evidence)

Recommendation 3. Use the severity of illness score (APACHE II) and other risk factors outlined above to plan appropriate medical or surgical therapy, and for evaluating the efficacy of different antimicrobial regimens for complicated IAIs (A-2 evidence)

\section{Key recommendations for microbiology and antimicrobial} susceptibility testing

Recommendation 4. Due to the predominance of certain virulent pathogens in IAIs, the concept of 'core' pathogens is recommended for planning initial empirical antimicrobial therapy (A-2 evidence).

Recommendation 5. The microbiology of community-acquired IAIs in the absence of previous antimicrobial exposure generally consists of 'core' pathogens that are readily predictable (A-2 evidence). In such patients and particularly those with mild to moderate severity of illness, routine bacteriological cultures of abdominal fluid or pus and antibiotic susceptibility testing of intra-abdominal isolates are optional and not routinely required to guide empirical antimicrobial therapy. However, such cultures may be useful for ongoing surveillance studies and generating local epidemiological data regarding antimicrobial susceptibility profiles and emerging resistance (A-2 evidence).

Recommendation 6. Patients with health care-associated IAIs who have prolonged previous hospitalization (five days or more), are severely ill (APACHE II score of 15 or greater) or have received previous antimicrobial therapy (more than two days) are at a greater risk for antimicrobial-resistant pathogens. In

${ }^{1}$ Division of Infectious Disease, Department of Medicine, University of British Columbia and Vancouver Hospital and Health Sciences Centre, Vancouver, British Columbia; ${ }^{2}$ Division of Infectious Diseases, Department of Medicine, Queen's University, Kingston; ${ }^{3}$ Department of Surgery, University of Toronto, Toronto, Ontario; ${ }^{4}$ Department of Surgery, University of Calgary, Calgary, Alberta; ${ }^{5}$ Departments of Pathology and Laboratory Medicine, University of Minnesota and Hennepin County Medical Center, Minnesota, USA; ${ }^{6}$ Department of Medical Microbiology and Medicine, University of Manitoba, Winnipeg, Manitoba; ${ }^{7}$ Department of Infectious Diseases and Microbiology, Hôspital Maisonneuve-Rosemont, University of Montreal, Montreal, Quebec

Endorsed by the Association of Medical Microbiology and Infectious Disease (AMMI) Canada and the Canadian Association of General Surgeons (CAGS) Committee on Acute Care Surgery and Critical Care

Correspondence: Dr Anthony W Chow, Division of Infectious Disease, Department of Medicine, University of British Columbia, 769 Burley Place, West Vancouver, British Columbia V7T 2A2. Telephone 604 926-4770, fax 604 926-4770, e-mail tonychow@interchange.ubc.ca 
such patients, blood and intraoperative cultures as well as antimicrobial susceptibility testing of all bacterial isolates should be performed routinely (A-2 evidence).

Recommendation 7. For specimen collection, abdominal fluid or pus should be collected in a capped airless syringe or be directly inoculated into appropriate aerobic and anaerobic transport media. Cultures should be sent for Gram stain and susceptibility testing. Swab specimens are not recommended (B-2 evidence).

Recommendation 8. In patients who develop treatment failures, their intra-abdominal cultures at reoperation are more likely to contain antibiotic-resistant isolates including nonfermenters and Candida species (A-2 evidence). Routine cultures and antimicrobial susceptibility testing of all isolates should be performed to guide subsequent antimicrobial therapy (A-2 evidence).

\section{Key recommendations for initial empirical antimicrobial therapy}

Recommendation 9. For patients with community-acquired IAIs with mild to moderate severity (APACHE II score lower than 15) who have not undergone prolonged previous hospitalization (five days or more) or received previous antimicrobial therapy (more than two days), initial empirical antimicrobial therapy should be directed against 'core pathogens' only, including enteric Gram-positive cocci as well as facultative and anaerobic Gram-negative bacilli, particularly Escherichia coli and Bacteroides fragilis (A-1 evidence). For adult patients, monotherapy with cefoxitin, ticarcillin-clavulanate, ertapenem, moxifloxacin or tigecycline is appropriate; alternatively, combinations of cefuroxime, cefotaxime, ceftriaxone or ciprofloxacin, each with metronidazole, are preferable to broader-spectrum regimens (A-1 evidence).

Recommendation 10. Ampicillin-sulbactam (not available in Canada), cefotetan and clindamycin are no longer recommended for routine empirical therapy of complicated IAIs because of the high rate of resistance among communityacquired E coli against ampicillin-sulbactam, and among B fragilis against cefotetan and clindamycin (B-2 evidence).

Recommendation 11. In light of the availability of less toxic regimens and unfavourable clinical response rates in randomized clinical trials, aminoglycosides are not recommended for routine empirical treatment of complicated IAIs (A-1 evidence).

Recommendation 12. For health care-associated surgical IAIs and seriously ill patients with community-acquired infections (APACHE II score of 15 or greater, previous hospitalization of five days or more, or previous antimicrobial therapy of two days or more), antimicrobial agents with broader spectrum of activity against facultative and anaerobic Gram-negative bacilli are recommended (B-2 evidence). For adult patients, monotherapy with piperacillin-tazobactam, imipenem-cilastatin, meropenem, or combinations of ceftazidime, cefepime or ciprofloxacin with metronidazole, or tigecycline in combination with ciprofloxacin are appropriate (B-2 evidence).

Recommendation 13. Intraveous (IV) to oral (PO) sequential treatment with a fluoroquinolone (such as monotherapy with moxifloxacin or combination therapy with ciprofloxacin plus metronidazole) may be a cost-effective alternative
(B-2 evidence). Continued surveillance for emerging resistance, particularly against facultative Gram-negative bacilli, should be implemented and periodic review of their efficacy and safety should be considered when choosing monotherapy or combination therapy with a fluoroquinolone (A-1 evidence).

Recommendation 14. In light of the emerging concern of extended-spectrum beta-lactamase (ESBL)-producing Enterobacteriaceae species due to selection pressure by increased use of oxyimino-cephalosporins (ceftazidime, ceftriaxone and cefotaxime), as well as ampC-producing nosocomial pathogens (resistant to all cephalosporins), the prolonged use of all cephalosporins in the health care setting is actively discouraged in favour of beta-lactam-beta-lactamase inhibitors or carbapenems (A-2 evidence).

Recommendation 15. Routine coverage for enterococci is not recommended in patients with community-acquired IAIs of mild to moderate severity (A-1 evidence). However, empirical antienterococcal therapy should be considered for immunosuppressed patients with health care-associated, postoperative or recurrent IAIs, those with antimicrobial exposure to cephalosporins and other broad-spectrum regimens selecting for enterococci, and those with valvular heart disease or intravascular prosthetic devices (B-3 evidence).

Recommendation 16. Coverage for Pseudomonas aeruginosa should be considered if it is the only pathogen recovered, if it is isolated from blood cultures, or if the patient has not responded to antimicrobial treatment that does not cover $P$ aeruginosa in the setting of health care-associated IAIs (B-2 evidence).

Recommendation 17. Anti-methicillin-resistant Staphylococcus aureus (MRSA) therapy should be administered for health care-associated IAIs in patients who are known to be colonized with the organism or have a history of MRSA infection. Vancomycin remains the agent of choice, although linezolid, daptomycin, tigecycline and quinupristin-dalfopristin may also be used. Vancomycin may also be considered for surgical prophylaxis in patients who are known to be MRSA carriers or if they come from facilities with a high prevalence of MRSA infection (B-2 evidence).

Recommendation 18. Targeted antifungal therapy is recommended for patients with severe community-acquired or nosocomial IAIs only if Candida species is isolated from intraabdominal or blood cultures. Fluconazole is the agent of choice if Candida albicans is isolated. For non-albicans Candida species, either an echinocandin (such as caspofungin, micafungin or anidulafungin) or a triazole (such as voriconazole) to which the organism is susceptible may be considered (B-2 evidence).

Recommendation 19. Amphotericin B is not recommended as initial therapy because of its toxicity (B-2 evidence).

Recommendation 20. Pre-emptive antifungal therapy with fluconazole or an echinocandin may be considered for seriously ill patients with a high risk for invasive candidiasis (eg, immunosuppression, postoperative or recurrent peritonitis, Candida colonization at multiple sites, etc); however, such a strategy has not been shown to impact mortality (C-2 evidence).

Recommendation 21. The duration of antimicrobial therapy should be guided by intraoperative findings and clinical response as assessed by resolution of fever and leukocytosis, 
abdominal examination and gastrointestinal function, and should be no more than five to seven days, unless it is difficult to achieve adequate source control (B-3 evidence).

Recommendation 22. Patients who continue to exhibit clinical evidence of infection at the end of seven days of antimicrobial therapy should be evaluated for residual infection, resistant microorganisms and other possible causes of treatment failure, rather than simply prolonging or broadening antimicrobial therapy (C-3 evidence).

Recommendation 23. In patients with postoperative or other health care-associated infections and those with clinical treatment failure, the acquisition or selection of resistant microorganisms should be strongly suspected, and further antimicrobial therapy should be guided by intraoperative cultures and susceptibility testing obtained directly from abscess fluid or the peritoneal cavity (B-2 evidence).

Recommendation 24. Development of clinical pathways (ie, a protocol approach) for the management of complicated IAIs based on local epidemiology of antimicrobial utilization and antibiotic resistance profiles is highly recommended. Such locally developed clinical pathways should standardize the approach to diagnosis, microbiological and radiological investigations, empirical antimicrobial therapy as well as policies regarding discharge and outpatient management. Such local guidelines should be established by a multidisciplinary team including surgeons, infectious disease and medical microbiology specialists, emergency physicians and other health care providers, and should reflect local resources and local standards of care (B-3 evidence).

Recommendation 25. Each institution should develop its own suite of performance measures to provide ongoing evaluation of the effectiveness and appropriateness of the local clinical pathways for complicated IAIs, ultimately leading to improved quality of care (B-3 evidence).

\section{Key recommendations for source control}

Recommendation 26. Adequate source control is the primary means of managing IAIs and should not be subjugated to antimicrobial therapy (A-2 evidence).

Recommendation 27. Operative approaches to source control should be used when it is necessary to resect a gangrenous or perforated viscus, patch a perforated viscus, divert the enteric stream or when percutaneous approaches to abscess drainage are not possible or have not been effective (A-3 evidence).

Recommendation 28. Small abscesses (less than $3 \mathrm{~cm}$ ) might be amenable to antimicrobial therapy without drainage (B-2 evidence).

Recommendation 29. In select patients, source control of perforated diverticulitis may be achieved by laparoscopic lavage and drainage (C-2 evidence).

Recommendation 30. In select patients, source control of infected pancreatic necrosis may be achieved using percutaneous approaches (B-2 evidence).

\section{Key recommendations for IAH in IAIs}

Recommendation 31. The presence of risk factors that may predispose to the development of IAH or ACS should be assessed in all acutely ill patients (APACHE II score greater than 15) with complicated IAIs (B-2 evidence).

Recommendation 32. Baseline intra-abdominal pressure (IAP) measurements should be determined in all critically ill patients with complicated IAIs if two or more risk factors for IAH or ACS are present (B-2 evidence). If IAH (IAP greater than $12 \mathrm{mmHg}$ ) or ACP (IAP greater than $20 \mathrm{mmHg}$ ) is present, serial IAP measurements should be performed throughout the patient's critical illness (A-3 evidence).

Recommendation 33. Surgical decompression should be considered for patients with refractory IAH or evidence of ACS. In addition, medical approaches to reduce IAP and associated end-organ dysfunction should be implemented, consistent with local practices in the care of critically ill patients (B-2 evidence).

\section{Key recommendations for infection control and prevention}

Recommendation 34. General measures important for reducing the risk of surgical site infections, such as avoiding hyperglycemia perioperatively, cessation of tobacco use at least 30 days before elective surgery, instructing the patient to shower with an antiseptic agent the night before the surgical procedure, etc, should be instituted in all patients undergoing intra-abdominal surgery (B-2 evidence). Hair removal is indicated only in cases in which the hair may hamper the surgical procedure itself. If hair has to be removed, it should be performed immediately before the surgery using electric clippers (A-1 evidence).

Recommendation 35. Surgical team members should adopt a recommended scrubbing procedure for at least $2 \mathrm{~min}$, including hands, arms and elbows (A-2 evidence).

Recommendation 36. A two-filter system installed in series should be in place in the operating room to ensure a clean environment, and air should enter the operating room through the ceiling and exhaust near the floor (B-2 evidence). Regular check-up of all physical parameters of the operating room and a complete maintenance program should be instituted at the local level (B-3 evidence).

Recommendation 37. Antibiotics for surgical prophylaxis should be used only if evidence from clinical trials is available, and in situations for which a surgical site infection may have major consequences (A-1 evidence). If surgical prophylaxis is to be administered, both the timing and dosing of the antibiotic infusion should be adjusted to attain peak tissue concentrations at the moment of incision and throughout surgery (A-1 evidence). In cases of prolonged surgical procedures, prophylactic antibiotics may need to be readministered intraoperatively (B-2 evidence).

Recommendation 38. The duration of antimicrobial therapy for the purpose of surgical prophylaxis in the absence of established infection should be limited to $24 \mathrm{~h}$ or less in patients with penetrating bowel trauma repaired within $12 \mathrm{~h}$, intraoperative contamination by enteric contents or nonperforating appendicitis in the absence of abscess or local peritonitis (A-1 evidence).

Recommendation 39. A hospital-wide surgical site infection surveillance program with continuous collaboration and feedback with the surgical team should be implemented to reduce surgical site infections (A-1 evidence).

Recommendation 40. To effectively control the spread of antibiotic-resistant organisms, an effective infection control program coupled with a rigorous antibiotic stewardship program should be implemented locally (A-1 evidence). 


\section{TABLE OF CONTENTS}

1. Introduction

2. Methodology

3. Epidemiology

3.1. Definition and classification of IAIs

3.2. Incidence and mortality

3.3. Risk stratification for poor outcomes

3.4. Key recommendations

4. Microbiology and Antimicrobial Resistance

4.1 Normal flora of the gastrointestinal tract

4.2 Microbial causes of IAIs

4.3 Proper specimen collection and handling

4.4 Antimicrobial activity against intra-abdominal pathogens

4.5 Increasing antimicrobial resistance among intra-abdominal isolates

4.6 Microbiology of treatment failures

4.7 Key recommendations

5. Antimicrobial Therapy

5.1. Determinants of antimicrobial therapy

5.1.1 Host factors

5.1.2 Microbial factors

5.2. Antimicrobial regimens in randomized clinical trials for IAIs

5.3. Initial empirical antimicrobial therapy

5.3.1. Mild to moderately severe community-acquired IAIs

5.3.2. Health care-associated or severe community-acquired IAIs

5.3.3. Empirical anti-MRSA therapy

5.3.4. Empirical antienterococcal therapy

5.3.5. Pre-emptive antifungal therapy

5.4. Duration of antimicrobial therapy

5.5. Management of the nonresponsive patient

5.6. Key recommendations

6. Source Control

6.1. Approaches to source control

6.2. Key recommendations

7. IAH and ACS

7.1. Definitions and pathophysiology

7.2. IAH and complicated IAIs

7.3. Management of IAH and ACS in complicated IAIs

7.4. Key recommendations for IAH in IAIs

8. Infection Control and Prevention

8.1. Patient-related issues

8.2. The operating room and related issues

8.3. Antimicrobial prophylaxis for surgical site infections

8.4. Prevention of the spread of antibiotic-resistant microorganisms

8.5. Key recommendations

9. Unanswered Questions and Future Directions

10. Tables

11. Figures and Figure Legends

12. Acknowledgements and Disclosures

13. Appendixes

14. References

\section{INTRODUCTION}

IAIs remain a major challenge in clinical practice. They are the main cause of postoperative morbidity following abdominal surgery and the most frequent cause for admission to a surgical intensive care unit $(2,3)$. IAIs differ from infections encountered elsewhere in several respects. First, the clinical spectrum of IAI is extremely wide, ranging from uncomplicated acute appendicitis with a relatively benign course to diffuse peritonitis from perforated viscus or ischemic bowel with high morbidity and mortality. While both scenarios comprise of IAIs, they require different approaches to diagnosis and treatment. Additionally, the role of surgery in the management of patients with IAIs is pivotal and generally considered to be a decisive factor in the outcome. The clinical and microbiological diagnosis is also often problematic: IAIs are typically polymicrobial, and not every microorganism involved can be identified in the clinical microbiology laboratory by routine cultures; the pathogenicity of certain microorganisms cultured from IAIs is not considered to be the same for every patient and often relates more directly to the severity of underlying disease or comorbid conditions of the host; and the clinical signs and symptoms do not often match the severity of disease and may lead to substantial delays in appropriate diagnosis and management (3-8). Additionally, antibiotic resistance among enteric pathogens has evolved globally and at an alarming rate, while very few newer agents have emerged to replace older therapeutic regimens.

The current clinical practice guideline was jointly developed by the CSS and AMMI Canada. The primary goal was to develop updated recommendations for the medical and surgical management of complicated IAIs since publication of the 2003 antimicrobial treatment guideline by the IDSA (1). Particular focus is directed at risk stratification for poor outcomes based on epidemiological studies, current status of antimicrobial susceptibility and resistance profiles among enteric pathogens, therapeutic efficacy of antimicrobial regimens based on randomized clinical trials, operative versus percutaneous approaches for source control, the role of IAH and ACS in IAIs, and infection control and preventive measures for postoperative IAIs and surgical site infections. An additional objective is to categorize the recommendations according to the strength and quality of the available evidence using a standardized grading system. Importantly, the current guideline provides recommendations for initial empirical antimicrobial management of complicated IAIs based on clinical settings and issues unique to the Canadian health care system (eg, publicly funded health care system and regionalization of health care delivery).

\section{METHODOLOGY}

These guidelines were prepared by a working group comprised of individuals with expertise in the disciplines of infectious disease, medical microbiology, general surgery, intensive care and pharmacy. Members were chosen based on their expertise and recommendations by the co-chairs of the Guidelines Committee who represent the professional societies of AMMI Canada and the CSS. Each member of the working group was responsible for specific sections of the guideline in accordance with their clinical knowledge, practice and expertise. The final document was derived from these individual contributions and edited by the co-chairs for organization, flow and consistency in style. The Medline database was searched for articles published 
in the English language between 1980 and May 2008. The general search strategy included 26 primary search terms including the following: "abdominal", "abscess", "acute pancreatitis", "anaerobes", "appendicitis", "cholecystitis", "intraabdominal", "infection", "necrotizing pancreatitis", "pancreatitis", "sepsis", "surgery", "abdominal compartment syndrome", "intra-abdominal hypertension" and "risk factors". Additional search terms such as "cephalosporins" or "tertiary" were further paired with words and phrases indicating an IAI (such as "tertiary peritonitis", "intra-abdominal infection" or "intra-abdominal sepsis"). Review was limited to randomized clinical trials in adults. Inclusion of antimicrobial agents was limited to agents currently approved by Health Canada or the Federal Food and Drug Administration of the United States. Reports from meta-analyses, practice guidelines, clinical conferences and major reviews were also examined. In addition, the Cochrane database on antibiotic regimens for secondary peritonitis of gastrointestinal origin in adults, published in 2006 (9), was searched to ensure that all prospective trials were included. Citations were imported into Reference Manager Software (Professional Edition, version 10, ISI ResearchSoft Inc, USA) for sorting, retrieval and in-depth analysis. Studies that were nonrandomized, had fewer than 25 evaluable patients in either study arm or represented duplicate publications were excluded. Outcome measures assessed were clinical success rates from evaluable patients and mortality from infection, unless otherwise specified. These studies form the basis of therapeutic and management recommendations, which were further categorized based on study design and quality according to the IDSA Public Health Service grading system for rating recommendations in clinical guidelines (10) (Table 1). Consensus was achieved using a Delphi process (11).

\section{Contributions and approval process}

Generation of the idea to develop the guideline was a group effort, facilitated by GAE in his role as Chairman of the AMMI Canada Clinical Guidelines Committee. Writing of the first draft was undertaken by AWC and GH, with input by all members of the panel. Review was undertaken by the whole group, with consensus achieved using a Delphi process. The final draft underwent extensive review, both internally by members of the AMMI Canada Clinical Guidelines Committee and the CSS, and externally by experts in the field. The final version was approved by the AMMI Canada Clinical Practice Guidelines Committee and endorsed by the Canadian Association of General Surgeons Committee on Acute Care Surgery and Critical Care.

\section{Disclosures}

All members of the Working Group complied with the AMMI Canada and CSS policy on conflicts of interest, which requires disclosure of any financial or other interests that might be construed as constituting an actual, potential or apparent conflict. Members of the Working Group were provided a conflict of interest disclosure statement and were asked to identify any affiliations or financial interests with pharmaceutical companies that might potentially be affected by the guideline. Information was requested regarding ownership of stock or stock options, employment or paid consultancy within the past two years, honoraria,
TABLE 1

Infectious Diseases Society of America Public Health Service grading system for rating recommendations in clinical guidelines (10)

\begin{tabular}{ll}
\hline $\begin{array}{l}\text { Rating } \\
\text { category }\end{array}$ & $\begin{array}{c}\text { Definition } \\
\text { (strength of recommendation) }\end{array}$ \\
\hline A & Strong support of a recommendation for or against use \\
B & Moderate support of a recommendation for or against use \\
C & Weak support of a recommendation for or against use \\
Grade (quality of evidence) \\
\hline 1 & Evidence from at least 1 properly randomized controlled trial \\
2 & Evidence from at least 1 well-designed clinical trial without \\
& randomization, from cohort or case-controlled studies, or from \\
& dramatic results from uncontrolled experiments \\
3 & Evidence from opinions of respected authorities, descriptive \\
& studies or reports of expert committees \\
\hline
\end{tabular}

speaker fees, educational grants and travel assistance to attend meetings. No potential conflicts were identified.

\section{EPIDEMIOLOGY}

\subsection{Definitions and classification of IAIs}

From a clinical viewpoint, two major types of IAI can be distinguished: uncomplicated and complicated. In uncomplicated IAIs, the infectious process only involves a single organ and no anatomical disruption is present. Generally, patients with such infections can be managed with surgical resection alone and no antimicrobial therapy besides perioperative prophylaxis is necessary. In complicated IAIs, the infectious process proceeds beyond the organ that is the source of the infection, and causes either localized peritonitis (often referred to as abdominal abscess) or diffuse peritonitis, depending on the ability of the host to contain the process within a part of the abdominal cavity $(1,4,5,12,13)$. Complicated IAIs usually require an invasive surgical procedure for source control (hence, also known as 'surgical IAI').

IAIs can be further classified as 'community acquired' or 'health care associated'. Community-acquired IAIs involve conditions such as gastroduodenal perforation, ascending cholangitis, cholecystitis, appendicitis or diverticulitis with or without perforation, and pancreatitis without previous surgical intervention or hospitalization $(14,15)$. Health care-associated IAI is defined as an infectious process that is absent at the time of hospital admission, but becomes evident at $48 \mathrm{~h}$ or more after admission, and includes anastomotic leaks and perforations as well as abscesses that develop as a complication of surgery $(3,4,16,17)$. Health care-associated IAI also includes infections acquired during the course of receiving treatment for other conditions in a health care setting, including the nursing home, dialysis unit or surgical day care, within the previous 12 months (18).

Peritonitis associated with IAI can be classified as primary, secondary or tertiary depending on the clinical presentation. Primary bacterial peritonitis is typically defined as a group of diseases with different causes, having in common only an infection in the peritoneal cavity without an obvious source of peritoneal contamination, such as in patients with chronic liver disease and ascites and those undergoing peritoneal dialysis $(8,19,20)$. Secondary peritonitis refers to infections that arise from microbes in the alimentary tract due to perforation of a hollow viscus causing contamination of the otherwise sterile peritoneal cavity. 
Tertiary peritonitis has been an evolving term, but is generally regarded as an infection in those patients who require more than one surgical intervention for source control and can often be classified as recurrent or persistent infections of the peritoneal cavity $(3,6,17)$. Such patients commonly present with frequent septic episodes due to an exaggerated host inflammatory response (21).

\subsection{Incidence and mortality}

Several studies have attempted to clarify the incidence and mortality rates of IAIs within specific patient populations, including the anatomical site and nature of the surgical setting such as trauma versus nontrauma or duration of surgery. These rates vary greatly. For example, the overall prevalence of acute pancreatitis has been reported to vary from as low as $1 \%$ to as high as $80 \%$ of IAIs (22-26). Rates of postoperative IAIs are reported to vary by anatomical site, with the highest occurring after small bowel surgery (5.3\% to $10.6 \%)$, followed by colon surgery $(4.3 \%$ to $10.5 \%)$, gastric surgery ( $2.8 \%$ to $12.3 \%)$, liver/pancreas surgery ( $2.8 \%$ to $10.2 \%)$, exploratory laparotomy $(1.9 \%$ to $6.9 \%)$ and appendectomy $(1.3 \%$ to $3.1 \%)(27)$.

Despite the introduction of new surgical techniques, broadspectrum antimicrobials, as well as improved supportive care within surgical intensive care units, the overall mortality rates in complicated IAIs have remained high, approaching $25 \%$ in secondary peritonitis (28-30). Patients who develop tertiary peritonitis have an even higher mortality rate $(3,6,31)$. Depending on the cause and severity of illness, mortality rates of tertiary peritonitis are approximately twice as high as those with secondary peritonitis, ranging from $30 \%$ to $64 \%(3,8)$. Dellinger et al (32) and others (33) showed that mortality in IAIs was more closely related to the severity of illness and associated organ failure than the origin or site of infection. Importantly, multiple studies (34-36) have demonstrated the adverse effect of inappropriate antimicrobial therapy on overall mortality of complicated IAIs.

\subsection{Risk stratification for poor outcomes}

Regardless of antimicrobial therapy, patients can be stratified into different risk groups for mortality based on predictable clinical parameters and comorbid conditions: 'low' (less than $5 \%$ mortality), 'moderate' (5\% to $15 \%$ mortality) and 'high' (15\% to 30\% mortality) (37). The APACHE II scoring system has been extensively validated for assessing severity of illness and predicting mortality in patients with complicated IAIs $(38,39)$. Patients can be stratified into those with mild to moderate severity (APACHE II score lower than 15) and highseverity (APACHE II score of 15 or greater) illness. These indexes may be particularly useful in planning appropriate medical or surgical therapy, and for evaluating the therapeutic efficacy of different antimicrobial regimens for complicated IAIs. Apart from the severity of illness, other prognostic factors include older age, hypoalbuminemia, prolonged hospitalization and previous antibiotic exposure (37). Such patients have a more variable clinical course, and are more likely to harbour health care-associated multiresistant pathogens (15). Conversely, patients with low or moderate APACHE II scores (10 or lower) have a more predictable microflora and favourable clinical course $(40,41)$.
Risk factors that predict treatment failure in IAI are more variable. Traditionally, local factors, such as the degree of peritoneal contamination and surgical technique, have been regarded as important predictors for surgical site infection and postoperative wound dehiscence $(42,43)$. More recent studies $(44,45)$ have focused on systemic factors and those known to affect tissue healing such as old age, smoking, malnutrition, diabetes, cardiovascular or lung disease, male sex, degree of blood loss and the operation itself as well as the inability to obtain source control as playing a significant role in the outcome of IAI. Multivariate analyses have identified a number of risk factors that largely relate to the patients' underlying physiological status, including a low serum albumin concentration, pre-existing medical disorders such as significant cardiovascular disease, and severity of illness as determined by high APACHE II scores (12,20,32,37,46-49). Taken collectively, these studies have revealed that the overall severity of illness (as determined by a high APACHE II score), receipt of inactive antimicrobial therapy, and the inability to achieve adequate source control with the initial operative procedure are the strongest prognosticators for mortality and poor outcome in complicated IAIs $(12,46,48,50,51)$. Additionally, certain underlying diseases and comorbid conditions such as diabetes, obesity, smoking and malnutrition, have been shown to play an important role in increasing the risk of surgical site infections. The role of corticosteroids on surgical site infections remains controversial. Some authors have reported an increased risk of surgical site infections in patients receiving immunosuppressive therapy, but others did not find any significant relationship. The influence of microbiological findings on prognosis is seldom mentioned. However, Christou et al (52) demonstrated that IAI treatment failure was significantly correlated with the presence of resistant microorganisms at the time of reoperation and that resistant Gram-negative organisms, such as $P$ aeruginosa, are more commonly encountered in high-risk patients.

\subsection{Key recommendations for risk assessment and stratification}

Recommendation 1. Categorize the severity of illness by using the APACHE II score according to low-moderate (lower than 15) or high (15 or greater) (A-2 evidence). Although the APACHE II scoring is infrequently used clinically outside of the critical care setting at present, it is recommended that physicians and surgeons consider introducing it into clinical use in patients with IAIs. A user-friendly APACHE II calculator can be found on the Web site <http://www.globalrph.com/ apacheii.htm>.

Recommendation 2. Identify high-risk patients for poor outcome by stratification according to community-acquired versus health care-associated IAIs, previous antibiotic exposure, and underlying comorbid conditions such as diabetes, severe cardiopulmonary disease or immunosuppression (A-2 evidence).

Recommendation 3. Use the severity of illness score (APACHE II) and other risk factors outlined above to plan appropriate medical or surgical therapy, and for evaluating the efficacy of different antimicrobial regimens for complicated IAIs (A-2 evidence). 
TABLE 2

Microbial flora in the gastrointestinal tract

\begin{tabular}{|c|c|c|}
\hline $\begin{array}{l}\text { Anatomical } \\
\text { site }\end{array}$ & $\begin{array}{l}\text { Inoculum } \\
\text { (cfu/mL or g) }\end{array}$ & Organisms \\
\hline Stomach & $0-$ few & Lactobacillus species \\
\hline $\begin{array}{l}\text { Duodenum, } \\
\text { jejunum }\end{array}$ & $0-\geq 10^{6}$ & $\begin{array}{l}\text { Streptococcus species, Lactobacillus } \\
\text { species, Enterobacteriaceae }\end{array}$ \\
\hline lleum & $10^{6}-10^{7}$ & $\begin{array}{l}\text { Streptococcus species, Lactobacillus } \\
\text { species, Enterobacteriaceae, } \\
\text { Bacteroides species }\end{array}$ \\
\hline \multirow[t]{4}{*}{ Colon } & $\begin{array}{l}\text { Dominant flora } \\
10^{9}-10^{11}\end{array}$ & $\begin{array}{l}\text { Bacteroides species, Prevotella species, } \\
\text { Eubacterium species, Bifidobacterium } \\
\text { species, Clostridium species, } \\
\text { Streptococcus species, Porphyromonas } \\
\text { species }\end{array}$ \\
\hline & $\begin{array}{l}\text { Subdominant flora } \\
10^{6}-10^{8}\end{array}$ & $\begin{array}{l}\text { Enterobacteriaceae (mostly Escherichia } \\
\text { coli), Streptococcus species, Enterococcus } \\
\text { species, Lactobacillus species }\end{array}$ \\
\hline & $\begin{array}{l}\text { Sparse flora } \\
<10^{6}\end{array}$ & $\begin{array}{l}\text { Enterobacteriaceae (non-E coli), } \\
\text { Klebsiella species, Citrobacter species, } \\
\text { Proteus species, Enterobacter species }\end{array}$ \\
\hline & $\begin{array}{l}\text { Transient flora } \\
<10^{6}\end{array}$ & Pseudomonas species, Candida species \\
\hline
\end{tabular}

cfu Colony-forming units. Adapted from references 4, 78 and 79

\section{MICROBIOLOGY AND ANTIMICROBIAL RESISTANCE}

\subsection{Normal flora of the gastrointestinal tract}

The endogenous microbial flora of the human gastrointestinal tract is complex, consisting of hundreds of different facultative and anaerobic bacterial species. The density and composition of the normal flora depends on the anatomical location in the gastrointestinal tract (Table 2) (53-55). In the stomach, there are only a few organisms, but the numbers and variety of bacterial species progressively increase from the duodenum to the ileum. In the colon, the bacterial load is very high $\left(10^{9}\right.$ colonyforming units [cfu]/g to $10^{11} \mathrm{cfu} / \mathrm{g}$ ) with the dominant flora being obligate anaerobes, especially Bacteroides species, Clostridium species and nonspore-forming Gram-positive bacilli. The subdominant colonic flora represents a lower bacterial load $\left(10^{6} \mathrm{cfu} / \mathrm{g}\right.$ to $10^{8} \mathrm{cfu} / \mathrm{g}$ ), with $E$ coli being the predominant organism, followed by other Enterobacteriaceae species present in lower numbers. Exogenous flora, such as Pseudomonas species and Candida species, may appear transiently, especially after exposure to antimicrobials.

The endogenous microbial flora of the human gastrointestinal tract remains quite constant over time and is similar among different individuals. However, this flora is readily influenced by a variety of host and environmental factors, including diet, underlying disease, hospitalization, previous antimicrobial therapy and recent surgery (Table 3) (56-59). Thus, knowledge of the anatomical location of the primary source of infection, underlying comorbid conditions, and whether the infection is community or health care associated, are the critical factors in predicting the most likely pathogens and their antibiotic susceptibility profiles. This information is pivotal in the selection of initial empirical antimicrobial therapy.

\subsection{Microbial causes of IAIs}

In contrast to primary peritonitis associated with chronic liver disease or peritoneal dialysis that is usually caused by a single
TABLE 3

Effect of host factors on the composition of the intestinal microflora

\begin{tabular}{lll}
\hline Site & Host factor & Effect on intestinal flora \\
\hline $\begin{array}{l}\text { Proximal small } \\
\text { bowel }\end{array}$ & Achlorhydria & $\uparrow$ Escherichia coli, \\
& & Bacteroides fragilis \\
& Vagotomy and pyloroplasty & $\uparrow B$ fragilis, \\
& & Bifidobacterium \\
Mid and distal & Regional enteritis, blind loop, & 'Colonic flora' \\
small bowel & diverticulitis, irradiation, & \\
& obstruction & \\
Colon & Colonic resection with & $\downarrow$ Anaerobes and some \\
& ileostomy & aerobes \\
\hline
\end{tabular}

pathogen, secondary or tertiary peritonitis are generally polymicrobial in etiology. Up to 15 different bacterial species may be cultured intraoperatively from the infected peritoneal cavity (average of 2.7 aerobic and 7.4 anaerobic species isolated per specimen) (Table 4) (60). Anaerobic species generally predominate over facultative isolates $(15,30,61-74)$. The pathogenesis of polymicrobial infections associated with secondary or tertiary peritonitis is complex $(62,75,76)$ and presents unique challenges to the clinician. First, it is not always clear which constituent(s) of the complex microflora are the key pathogens following peritoneal contamination and which are simply symbionts or commensals. The numerical predominance of an organism within its natural ecological niche of the gastrointestinal tract does not necessarily imply greater pathogenicity or clinical significance. Thus, whereas E coli and encapsulated B fragilis constitute less than $5 \%$ of the total colonic microflora, nevertheless, they are recognized as the key pathogens in intra-abdominal sepsis and abscess formation $(60,75)$. Conversely, a highly virulent organism may be missed or overgrown in mixed culture due to its low density within the inoculum.

Due to the predominance of certain virulent pathogens and the polymicrobial nature of IAIs, the concept of 'core' pathogens was developed (Table 5). In community-acquired IAIs in which no previous antimicrobial exposure has occurred, the microbial causes of infection are relatively predictable and consist of the 'core' pathogens outlined in Table 5. These include anaerobes particularly B fragilis, nonfragilis Bacteroides species, Clostridium species, Fusobacterium species, Peptostreptococcus species, Lactobacillus species and Veillonella species. Facultative isolates include Streptococcus species, and Enterobacteriaceae species such as E coli, Klebsiella species, Enterobacter species, Proteus species and Serratia species. Although methicillin-sensitive $S$ aureus is commonly recovered from patients with IAIs, it is not a common pathogen in community-acquired IAIs (4,77-79).

\subsection{Proper specimen collection and handling}

The issue of whether routine intraperitoneal culture and antimicrobial susceptibility testing for obligate anaerobes should be performed for all patients with mild to moderately severe community-acquired surgical IAIs is controversial. As noted previously, the enteric microflora in patients with gangrenous or perforated appendicitis is complex. Routine cultures for these specimens are both time-consuming and costly (80). A number of studies (81-83) reported that routine performance of such cultures in mild to moderately severe community-acquired 


\section{TABLE 4}

Common microbial causes of intra-abdominal infections

\begin{tabular}{lc}
\hline Microbiological diagnosis & Frequency of isolation (\% of patients) \\
\hline Gram-negative bacilli & $50-100$ \\
Escherichia coli & \\
Klebsiella species & \\
Enterobacter species & \\
Proteus species & \\
Serratia marcescens & $10-44$ \\
Gram-positive cocci & \\
Streptococcus species & $48-92$ \\
Anaerobes & \\
Bacteroides fragilis & \\
Non-fragilis Bacteroides & \\
species & \\
Clostridium species & \\
Fusobacterium species & \\
Peptostreptococcus species & \\
Lactobacillus species & \\
Veillonella species &
\end{tabular}

IAIs has failed to demonstrate any beneficial impact on clinical outcome. Accordingly, routine culture of enteric contents from the peritoneal cavity in such patients may not be necessary (B-2 evidence). On the other hand, in patients with postoperative or other health care-associated infections and those with clinical treatment failure, the acquisition or selection of resistant microorganisms is more likely. In such patients, intraoperative cultures obtained directly from abscess fluid or the peritoneal cavity may be important for guiding therapeutic decisions and are strongly recommended $(60,84)$ (B-2 evidence).

\subsection{Antimicrobial activity against IAI pathogens}

The in vitro activity of commonly used antimicrobials against IAI pathogens including facultative Gram-positive cocci and Gram-negative bacilli and anaerobes are listed in Appendixes 1 to 3. These tables demonstrate that second-generation cephalosporins (eg, cefoxitin), third-generation cephalosporins (eg, cefotaxime, ceftriaxone, ceftazidime and cefepime), broadspectrum penicillins (eg, piperacillin/tazobactam and ticarcillin/ clavulanate), fluoroquinolones (eg, ciprofloxacin, levofloxacin and moxifloxacin), aminoglycosides (eg, gentamicin, netilmicin, tobramycin and amikacin), carbapenems (eg, imipenem, meropenem and ertapenem) and tigecycline have broad-spectrum activity against both Gram-positive cocci and Gram-negative bacilli commonly isolated in IAIs. Aminoglycosides such as gentamicin have very good activity against Gram-negative bacilli, but limited activity against Gram-positive cocci. Other agents, such as clindamycin, linezolid and vancomycin, have excellent activity against facultative Gram-positive cocci, but minimal activity against Gram-negative bacilli. The most active antimicrobials against anaerobes include metronidazole, carbapenems and broad-spectrum penicillins. Clindamycin has retained activity against most anaerobes, but resistance among $B$ fragilis and B fragilis group is escalating (85). Cefoxitin also exhibits decreased activity, particularly against the $B$ fragilis group organisms $(86,87)$. Tigecycline has excellent anaerobic activity including Peptostreptococcus species, B fragilis and B fragilis group organisms. Moxifloxacin is the most active fluoroquinolone against $B$ fragilis.
TABLE 5

'Core' pathogen concept in intra-abdominal infections

\begin{tabular}{|c|c|c|c|}
\hline \multirow[b]{2}{*}{ Infection } & \multicolumn{2}{|c|}{ Diagnostic } & \multirow[b]{2}{*}{ Likely pathogens } \\
\hline & Classification & features & \\
\hline $\begin{array}{l}\text { Community } \\
\text { acquired }\end{array}$ & Group 1 & $\begin{array}{l}\text { No previous } \\
\text { antimicrobial } \\
\text { use }^{*}\end{array}$ & 'Core' pathogens ${ }^{\dagger}$ \\
\hline $\begin{array}{l}\text { Community } \\
\text { acquired }\end{array}$ & Group 2 & $\begin{array}{l}\text { Previous } \\
\text { antimicrobial } \\
\text { use }^{\star}\end{array}$ & $\begin{array}{l}\text { 'Core' pathogens }{ }^{\dagger} \text { plus } \\
\text { resistant Gram-negative } \\
\text { bacilli, Enterococcus species, } \\
\text { Pseudomonas aeruginosa } \\
\text { and MRSA }\end{array}$ \\
\hline $\begin{array}{l}\text { Health care } \\
\text { associated }\end{array}$ & Group 3 & $\begin{array}{l}\text { With/without } \\
\text { previous } \\
\text { antimicrobial } \\
\text { use* }^{*}\end{array}$ & $\begin{array}{l}\text { 'Core' pathogens }{ }^{\dagger} \text { plus } \\
\text { resistant Gram-negative } \\
\text { bacilli, Enterococcus species, } \\
\text { P aeruginosa and MRSA }\end{array}$ \\
\hline
\end{tabular}

${ }^{*}$ Risk factors for antimicrobial-resistant pathogens include nosocomial infection and/or previous antimicrobial therapy in the past 90 days; ${ }^{\dagger}$ Core pathogens include Streptococcus species, Enterobacteriaceae (eg, Escherichia coli, Klebsiella species, Proteus species, Serratia marcescens) and anaerobes (eg, Bacteroides fragilis, non-fragilis Bacteroides species, Clostridium species, Fusobacterium species, Lactobacillus species, Peptostreptococcus species and Veillonella species). MRSA Methicillin-resistant Staphylococcus aureus

\subsection{Increasing antimicrobial resistance among intra-abdominal isolates}

Extensive surveillance studies (88-90) have demonstrated increasing antibiotic resistance globally among intra-abdominal isolates including B fragilis, B fragilis group species and Enterobacteriaceae species. For example, national surveys in the United States revealed that resistance to clindamycin among $B$ fragilis and $B$ fragilis group species has climbed steadily since 1997, reaching $19 \%$ and 26\%, respectively, in 2004 (85). Similarly, resistance to cefotetan among B fragilis group species has exceeded 40\% $(91,92)$. Accordingly, clindamycin and cefotetan are no longer recommended as empirical therapy for surgical IAIs. In addition, ampicillin-sulbactam (not marketed in Canada) is no longer recommended as routine empirical therapy for surgical IAIs due to widespread resistance of $E$ coli to this agent (88).

Patients with health care-associated infection or tertiary peritonitis are more likely to harbour resistant enteric Gramnegative bacilli (such as Klebsiella species, Enterobacter species, Serratia species, Acinetobacter species and Pseudomonas species, as well as other nonlactose-fermenting Gram-negative bacilli), facultative Gram-positive cocci (such as Enterococcus species and MRSA) and yeasts (particularly Candida albicans and Candida glabrata) $(15,90)$. Patients with postoperative IAIs and those with prolonged previous hospitalization (five days or more) or those who received previous antimicrobial therapy (more than two days) are particularly at risk for the acquisition or selection of resistant enteric pathogens (90). ESBL-producing Enterobacteriaceae species (10\% to 20\% of isolates) (89) and fluoroquinolone-resistant $E$ coli $(2 \%$ to $7 \%)(93,94)$ are of particular concern in such patients.

\subsection{Microbiology of treatment failures}

The microbiology associated with treatment failures has been documented in several randomized clinical trials (52,95-105). Cultures obtained from treatment failures are frequently polymicrobial and more likely to isolate E coli, Enterococcus species, $P$ aeruginosa and other nonfermenters. Furthermore, organisms 
isolated at reoperation for treatment failures are more likely to be resistant to the original treatment regimens (52).

\subsection{Key recommendations for microbiology and antimicrobial susceptibility testing}

Recommendation 4. Due to the predominance of certain virulent pathogens in IAIs, the concept of 'core' pathogens is recommended for planning initial empirical antimicrobial therapy (A-2 evidence).

Recommendation 5. The microbiology of community-acquired IAIs in the absence of previous antimicrobial exposure generally consists of 'core' pathogens that are readily predictable (A-2 evidence). In such patients and, particularly those with mild to moderate severity of illness, routine bacteriological cultures of abdominal fluid or pus and antibiotic susceptibility testing of intra-abdominal isolates are optional and not routinely required to guide empirical antimicrobial therapy. However, such cultures may be useful for ongoing surveillance studies and generating local epidemiological data regarding antimicrobial susceptibility profiles and emerging resistance (A-2 evidence).

Recommendation 6. Patients with health care-associated IAIs who have prolonged previous hospitalization (five days or more), are severely ill (APACHE II score of 15 or greater) or have received previous antimicrobial therapy (more than two days) are at greater risk for antimicrobial-resistant pathogens. In such patients, blood and intraoperative cultures as well as antimicrobial susceptibility testing of all bacterial isolates should be performed routinely (A-2 evidence).

Recommendation 7. For specimen collection, abdominal fluid or pus should be collected in a capped airless syringe or be directly inoculated into appropriate aerobic and anaerobic transport media. Cultures should be sent for Gram stain and susceptibility testing. Swab specimens are not recommended (B-2 evidence).

Recommendation 8. In patients who develop treatment failures, their intra-abdominal cultures at reoperation are more likely to contain antibiotic-resistant isolates including nonfermenters and Candida species (A-2 evidence). Routine cultures and antimicrobial susceptibility testing of all isolates should be performed to guide subsequent antimicrobial therapy (A-2 evidence).

\section{ANTIMICROBIAL THERAPY}

The selection of initial anti-infective agents for complicated IAI was previously reviewed in 2003 by the IDSA (1). However, a number of newer antimicrobial regimens have been prospectively evaluated for the treatment of IAI since then, particularly ciprofloxacin plus metronidazole as sequential therapy, and monotherapy with moxifloxacin or tigecycline. The current practice guideline aims to update the contemporary status of antimicrobial agents for the empirical treatment of surgical IAIs. A strategy of risk stratification based on severity of illness, hospital versus community acquisition, previous antimicrobial therapy and likelihood of resistant pathogens is proposed.

\subsection{Determinants of antimicrobial therapy}

5.1.1 Host factors: Complicated IAIs develop in the presence of impaired host defences either due to local trauma of abdominal organs or systemic immune dysfunction from comorbid disease, or both. The peritoneum mounts three major host defence mechanisms in response to infectious agents: bacterial clearance via the diaphragmatic lymphatics; phagocytic killing by resident peritoneal macrophages and recruited neutrophils; and sequestration processes, which involve $\mathrm{T}$ cell activation $(106,107)$, deposition of fibrin exudates (108) and walling off the infection. Should these local and systemic host defences fail to control the infection, diffuse peritonitis may ensue. Alternatively, if the acute inflammatory host response to infection is overwhelming, bacterial products and deleterious biological host response mediators (such as cytokines) lead to tissue hypoxia, irreversible shock, multiple organ system failure and, ultimately, death (21). Thus, host factors, particularly the balance between host defences and the systemic inflammatory response, are critical determinants of eventual outcome. Accordingly, effective antimicrobial therapy is only one component of the treatment goal. Timely hemodynamic resuscitation and support of vital organ function to prevent irreversible hypoperfusion and shock, and rapid anatomical diagnosis to institute adequate source control are just as important as appropriate antimicrobial therapy.

5.1.2 Microbial factors: IAIs that arise from the endogenous enteric flora are polymicrobial in nature $(1,4,79,109)$. The polymicrobial etiology of IAIs presents several dilemmas in clinical practice. For example, microorganisms in mixed infections may respond to antimicrobial agents differently than those in monomicrobial infections, and it may not be necessary to eradicate every bacterial species in mixed infections to achieve a cure. A case in point is the isolation of enterococci from intraperitoneal cultures in patients with mild to moderately severe community-acquired surgical IAIs. Despite the routine isolation of enterococci, treatment regimens providing antienterococcal coverage were not superior to comparative regimens that did not provide such coverage in prospective, blinded and randomized clinical trials $(1,110)$. However, in seriously ill or immunocompromised patients, and in patients with health care-associated infections, the isolation of enterococci is a risk factor for treatment failure and increased mortality; hence, empirical antienterococcal coverage in such patients appears warranted $(111,112)$. Similarly, isolation of $P$ aeruginosa in a polymicrobial infection does not necessarily require treatment because it may represent a transient flora. However, treatment should be considered when $P$ aeruginosa is the only pathogen recovered, if the patient is bacteremic, or if the patient has not responded to initial antimicrobial treatment that does not cover $P$ aeruginosa $(1,4,8,13,16,37,53,79,109,113,114)$. Thus, initial empirical antimicrobial therapy should be directed at the 'core' pathogens discussed earlier. Modification of therapy will be required depending on host factors such as severity of illness, underlying disease, whether health care associated or community acquired, and previous antimicrobial exposure.

\subsection{Antimicrobial regimens in randomized clinical trials for IAIs}

A total of 102 antimicrobial regimens have been adequately studied in randomized clinical trials for IAIs in adults over the 


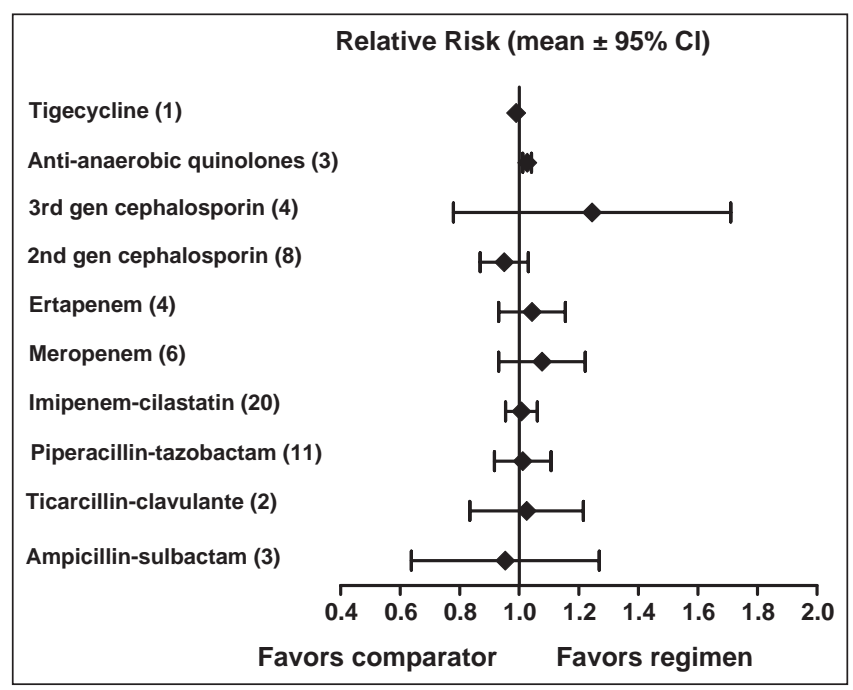

Figure 1) Ratio of clinical success rates over comparators in randomized controlled trials among 63 monotherapy regimens for complicated intra-abdominal infections. Data are expressed as mean $\pm 95 \%$ CIs of study regimens over the comparator in each trial. Number of studies for the antimicrobial groups are shown in parentheses. Secondgeneration (gen)cephalosporins include cefoxitin, cefotetan, cefuroxime and cefamandole. Third-gen cephalosporins include cefoperazone with/without sulbactam. Antianaerobic fluoroquinolones include moxifloxacin, clinafloxacin and trovafloxacin. Data were compiled from published results of prospective randomized comparative trials $(49,52,62,70,95,96,98,100-104,110,116-120,129,217-243)$

past two decades (Figures 1 and 2). These regimens were administered to 9900 evaluable patients, with an average of 97 evaluable patients per study regimen (range 26 to 631; median 65). These study regimens can be grouped into 63 monotherapy regimens with single agents (beta-lactam antibiotics with/without beta-lactamase inhibitors, carbapenems, fluoroquinolones, or glycylcycline [tigecycline]) (Figure 1), and 39 combination regimens with two or more agents active against facultative Gramnegative bacilli (aminoglycosides, cephalosporins, monobactam or fluoroquinolones) and anaerobes (clindamycin or metronidazole) (Figure 2). Among monotherapy regimens, imipenemcilastatin was most frequently studied (20 trials), followed by meropenem or ertapenem (10 trials), piperacillin with/without tazobactam (11 trials), second-generation cephalosporins (cefoxitin, cefotetan, cefuroxime, cefamandole; eight trials), ampicillin or ticarcillin plus beta-lactamase inhibitor (five trials), third-generation cephalosporins (cefoperazone with/ without sulbactam; four trials), fluoroquinolones (trovafloxacin, clinafloxacin, moxifloxacin; three trials) and tigecycline (pooled analysis of two trials). Among the combination regimens, aminoglycoside plus clindamycin was most frequent (19 trials), followed by cephalosporin (cefuroxime, cefotaxime, ceftriaxone, cefoperazone or cefepime) plus metronidazole (11 trials) or clindamycin (one trial), fluoroquinolone plus metronidazole (five trials), aztreonam plus clindamycin (two trials) and piperacillintazobactam plus amikacin (one trial). Three randomized clinical trials evaluating two new monotherapy regimens were included. One of these evaluated the efficacy, safety and cost-savings of moxifloxacin as sequential therapy compared with piperacillin-tazobactam in both community- and health

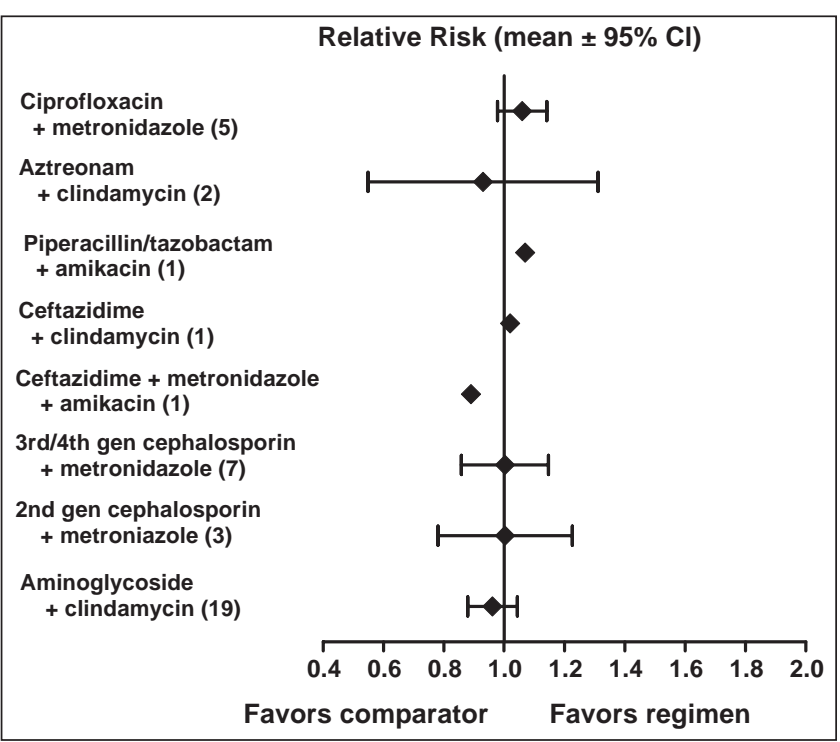

Figure 2) Ratio of clinical success rates over comparators in randomized controlled trials among 39 combination regimens for complicated intra-abdominal infections. Data are expressed as mean $\pm 95 \%$ CIs of study regimens over the comparator in each trial. Number of studies for the antimicrobial groups are shown in parentheses. Second-generation (gen) cephalosporins include cefuroxime. Third-or fourth-gen cephalosporins include cefotaxime, ceftriaxone and cefepime. Data were compiled from published results of prospective, randomized comparative trials $(49,62,70,95,100,103,104,110,117-120,127,218,219,223$ $226,229-232,237-247)$

care-associated complicated IAIs (102). The other is a pooled analysis of two phase 3, double-blind, randomized trials evaluating the efficacy and safety of tigecycline compared with imipenem-cilastatin $(98,115)$. Only one study (116) examined the duration of empirical antimicrobial therapy comparing the efficacy and safety of ertapenem $1 \mathrm{~g} /$ day for three days versus five days or more in a prospective, double-blind, multicentre trial. No statistically significant differences in outcome were identified.

Overall, the clinical outcome of all these regimens was favourable, with mean success rates ranging from $44 \%$ to $100 \%$ (mean $82 \%$, median $83 \%$ ), and infection-related mortality rates ranging from $0 \%$ to $17 \%$ (mean $3.7 \%$, median $2.5 \%)$ in the different study groups. However, the disease severity score of enrolled patients was generally low, with $48 \%$ to $90 \%$ (mean $77 \%$, median $82 \%$ ) of patients having an APACHE II score of 10 or lower, and mean APACHE II scores ranging from 5.5 to 13.1 (mean 8.0, median 7.8 ) in the different treatment groups. Furthermore, more than $80 \%$ of study populations had community-acquired infections. Among these 102 therapeutic regimens, only eight studies demonstrated a statistically superior clinical success rate of the study arm over its comparator $(49,62,96,110,117-120)$. Of interest, the aminoglycoside (gentamicin or tobramycin) plus clindamycin regimen was inferior to the comparator (cefoperazone-sulbactam and imipenem-cilastatin, respectively) in two separate prospective, randomized trials $(49,118)$. Conversely, cefoperazone was superior to the comparator in two separate prospective trials (ceftazidime/metronidazole/amikacin and gentamicin/clindamycin, respectively) $(62,118)$. However, 
TABLE 6

Antimicrobial agents for empirical therapy of communityacquired surgical intra-abdominal infections with low to moderate severity (APACHE II score lower than 15)

Monotherapy

\section{Cefoxitin 2 g IV q6h}

Ticarcillin-clavulanate $3 \mathrm{~g} / 0.1 \mathrm{~g}$ IV q4-6h

$\rightarrow$ Amoxicillin-clavulanate $500 \mathrm{mg} / 125 \mathrm{mg}$ PO q8h

Ertapenem $1 \mathrm{~g} \mathrm{IV}$ once daily*

Moxifloxacin $400 \mathrm{mg} \mathrm{IV} \rightarrow \mathrm{PO}$ once daily*

Tigecycline $100 \mathrm{mg}$ IV loading, then $50 \mathrm{mg}$ IV q12h

Combination

Second- or third-generation cephalosporin (cefuroxime $1.5 \mathrm{~g} \mathrm{IV} \mathrm{q8h,}$ cefotaxime $1.5 \mathrm{~g} \mathrm{IV} \mathrm{q8h}$ or ceftriaxone $1-2 \mathrm{~g} \mathrm{IV}$ once daily*) plus metronidazole $500 \mathrm{mg} \mathrm{IV} \rightarrow \mathrm{PO}$ q8h

Ciprofloxacin $200 \mathrm{mg} \mathrm{IV} \mathrm{q12h} \rightarrow 500 \mathrm{mg}$ PO q12h plus metronidazole $500 \mathrm{mg}$ IV $\rightarrow \mathrm{PO}$ q $8 \mathrm{~h}$

*Suitable for home or outpatient intravenous (IV) therapy. Exact dosing may require modification based on renal and/or hepatic function. IV $\rightarrow P O I V$ to oral sequential therapy; q Every

no single regimen was consistently superior to another regimen in more than one study. This is demonstrated by the similar relative risk values of clinical success rates (expressed as the ratio of the study regimen over the comparator in each trial) among the 63 monotherapy regimens (mean 1.02; 95\% CI 0.99 to 1.06 ) (Figure 1), and the 39 combination regimens (mean 0.99; 95\% CI 0.94 to 1.03 ) (Figure 2).

It should be noted that these published studies primarily enrolled patients with mild to moderately severe, communityacquired IAIs. Furthermore, these studies do not address the changing trend and local epidemiology of antibiotic resistance patterns of intra-abdominal isolates. In addition, although prospective, randomized clinical trials have been the gold standard for formulating therapeutic recommendations, the study populations enrolled in such trials may be vastly different from those not enrolled in a clinical trial. Study patients generally have a more favourable outcome due to less severe illness, infrequent association with antibiotic resistance, or exclusion of complex underlying diseases (121). Thus, conclusions drawn from prospective, randomized clinical trials may not be generally applicable to all patients with IAI in the 'real world' setting of clinical practice.

\subsection{Initial empirical antimicrobial therapy}

Based on the above discussion of microbial and host factors that predict outcome, and published studies of randomized clinical trials, it is recommended that the selection of initial antimicrobial therapy for surgical IAI be stratified according to the following risk factors: community- versus health careassociated infection, severity of illness as assessed by APACHE II scores ( 15 or lower for mild to moderately severe and greater than 15 for highly severe), and prolonged previous hospital stay (five or more days) or antimicrobial therapy (two or more days). Patients with prolonged previous hospitalization or antimicrobial exposure are more likely to harbour resistant microorganisms and should be treated as for health care-associated infections (90) (A-1 evidence). Empirical antimicrobial therapy should be initiated as soon as the diagnosis of IAI is suspected (preferably within $8 \mathrm{~h}$ of presentation to a health care facility) $(122,123)$.
TABLE 7

Antimicrobial agents for empirical therapy of health careassociated surgical intra-abdominal infections, or for severely ill patients (APACHE II score of 15 or greater) with community-acquired infection*

Monotherapy

Piperacillin-tazobactam 3.375 g IV q4-6h or 4.5 g IV q6-8h

Imipenem-cilastatin $0.5 \mathrm{~g}$ IV q6h or meropenem $0.5 \mathrm{~g} \mathrm{IV} \mathrm{q6h} \mathrm{to} 1 \mathrm{~g}$ IV q8h

\section{Combination}

Third- or fourth-generation cephalosporin (ceftazidime $2 \mathrm{~g}$ IV or cefepime $2 \mathrm{~g}$ IV q8h) plus metronidazole $500 \mathrm{mg}$ IV q8h

Ciprofloxacin $400 \mathrm{mg}$ IV q12h (q8h if for Pseudomonas aeruginosa) $\rightarrow$ $500 \mathrm{mg}$ PO bid, plus metronidazole $500 \mathrm{mg}$ q8h IV $\rightarrow$ PO

Tigecycline $100 \mathrm{mg}$ IV loading, then $50 \mathrm{mg}$ IV q12h plus ciprofloxacin $400 \mathrm{mg}$ IV q8h

Piperacillin-tazobactam $3.375 \mathrm{~g}$ IV q4-6h or $4.5 \mathrm{~g}$ IV q6-8h plus an aminoglycoside

Imipenem-cilastatin $0.5 \mathrm{~g}$ IV q6h or meropenem $0.5 \mathrm{~g}$ IV q6h to $1 \mathrm{~g} \mathrm{IV} \mathrm{q8h}$ plus an aminoglycoside

*Includes patients with prolonged previous hospitalization (five days or more) or previous antimicrobial therapy (two days or more). Exact dosing may require modification based on renal and/or hepatic function. bid Twice a day; IV Intravenous; PO Oral; q Every

Antimicrobial therapy is directed at a polymicrobial infection caused by 'core' pathogens including facultative enteric Gramnegative bacilli and intestinal anaerobes, particularly E coli and $B$ fragilis. For most mild to moderately severe communityacquired IAIs, the microbiology and antibiotic susceptibility profile of peritoneal isolates are predictable. Routine intraabdominal cultures and antibiotic susceptibility testing in such patients are optional and may not be cost-effective. Monotherapy with single agents possessing a relatively narrow spectrum of activity may suffice (Table 6). As noted earlier, ampicillinsulbactam, clindamycin and cefotetan are no longer recommended for routine empirical therapy of IAIs because of the high rate of resistance to ampicillin-sulbactam among $E$ coli, and to cefotetan and clindamycin among B fragilis.

For high-risk patients (those with health care-associated infection, previous antimicrobial therapy, severe physiological impairment [APACHE II score of 15 or greater] and those who failed to respond to initial treatment), an antimicrobial regimen with a broader spectrum of activity against resistant facultative Gram-negative rods as well as anaerobes is recommended (Table 7). In such patients, availability of intraoperative cultures from the peritoneal cavity and in vitro susceptibility testing of recovered isolates are critical to guide further adjustments in antimicrobial therapy. The selection of specific antimicrobial regimens is discussed below.

5.3.1. Mild to moderately severe community-acquired IAI: For patients with community-acquired infection of mild to moderate severity (APACHE II score lower than 15), an antibiotic regimen with a relatively narrow spectrum of activity, low toxicity profile and low cost is recommended. These include monotherapy with cefoxitin, a beta-lactam-beta-lactamase inhibitor (eg, ticarcillin-clavulanate), ertapenem, moxifloxacin or tigecycline, and combination regimens of a second- or thirdgeneration cephalosporin or ciprofloxacin, each with metronidazole (Table 6) (A-1 evidence). Combination therapy with ciprofloxacin plus metronidazole offers the additional 
benefit of IV to oral PO sequential therapy, enabling earlier discharge from hospital and potential cost savings to the health care system. Furthermore, the fluoroquinolones such as ciprofloxacin have enjoyed a long history of successful treatment for a variety of gastrointestinal and IAIs (124). Meta-analysis of several randomized trials in 1431 patients have demonstrated that the ciprofloxacin/metronidazole combination is at least as effective, if not superior, to beta-lactam-based regimens $(110,125$ 127). In one study by Cohn et al (110), the clinical success rate among 134 patients receiving ciprofloxacin/metronidazole was significantly higher than that among 116 patients who received piperacillin-tazobactam $(74 \%$ versus $63 \% ; \mathrm{P}<0.05)$. There was no difference in all-cause mortality or toxicity between ciprofloxacin/metronidazole and the comparison arms. However, the availability of ciprofloxacin/metronidazole as IV to PO sequential therapy offers considerable cost advantage because patients may be discharged from hospital several days earlier (128).

More recently, moxifloxacin once daily as sequential therapy has been shown to be equally effective as piperacillintazobactam IV fourtimesdaily followed by amoxicillin-clavulanate $\mathrm{PO}$ in community-acquired IAIs (clinical success rates, $80 \%$ versus $82 \%$ ) (102). In contrast to trovafloxacin and clinafloxacin, which were also comparable to imipenem-cilastatin in efficacy $(101,129)$, moxifloxacin did not exhibit severe adverse effects such as hepatotoxicity, hypoglycemia or phototoxicity. (Note: The European Medicines Agency [EMEA] released a recommendation to restrict the use of oral formulations of moxifloxacin-containing medicines in July 2008 after completing a review of new data related to significant hepatotoxicity $<$ http://www.ema.europa.eu/pdfs/human/referral/ moxifloxacin_107/Q\&A_Moxifloxacin_38045408en.pdf >.) It remains to be seen whether this fluoroquinolone will replace ciprofloxacin/metronidazole as a major therapeutic regimen in more seriously ill patients. In contrast to ciprofloxacin and moxifloxacin, there are no randomized clinical trials evaluating the use of levofloxacin or gatifloxacin in combination with metronidazole for complicated IAIs. One concern with the increased use of fluoroquinolones is the emergence of resistance among Enterobacteriaceae species, particularly ESBLproducing strains $(89,93)$. The rates of fluoroquinolone resistance from 1993 to 2004 among 74,394 Gram-negative bacilli isolated from intensive care unit patients in a large number of American acute care hospitals were compared (130). The per cent of $\mathrm{E}$ coli isolates resistant to ciprofloxacin rose from $0.9 \%$ from 1993 to 1995 , to $17.3 \%$ from 2002 to 2004, while that among $P$ aeruginosa rose from $11.2 \%$ to $28.9 \%$. The rate of fluoroquinolone resistance was directly linked to total fluoroquinolone usage. This relationship was also demonstrated by other studies $(131,132)$. Thus, continued vigilance through surveillance, and innovative approaches to minimize or delay the emergence of resistance, are necessary if the clinical utility of these agents as initial empirical therapy is to be preserved.

Although aminoglycoside plus an antianaerobic agent has been the gold standard against which newer agents were compared for more than a decade, this combination is no longer considered to be standard therapy even for mild to moderately severe community-acquired infections (A-2 evidence). This is primarily due to unacceptable nephrotoxicity and ototoxicity of aminoglycosides in the presence of alternative safer agents such as beta-lactam-beta-lactamase inhibitors, third- and fourth-generation cephalosporins and fluoroquinolones (133). More importantly, meta-analysis of clinical success rates comparing regimens using aminoglycoside plus an antianaerobic agent against all other regimens in the Cochrane database revealed significantly lower response rates of the former (OR 0.65, 95\% CI 0.46 to 0.92; $\mathrm{P}=0.02$ ) (9). In particular, the clinical success rates of imipenem-cilastatin and cefoperazonesulbactam (no longer available in Canada) were both superior to aminoglycoside/clindamycin regimens in two prospective, randomized trials by Solomkin et al (49) and Jauregui et al (118), respectively. The outcome of microbiological success was also significantly different in favour of the comparator regimens (OR 0.49; 95\% CI 0.31to 0.76; $\mathrm{P}=0.001$ ) (9).

In light of the emerging concern of ESBL-producing Enterobacteriaceae species due to selection pressure by increased use of oxyimino-cephalosporins (ceftazidime, ceftriaxone and cefotaxime), as well as ampC-producing nosocomial pathogens (resistant to all cephalosporins), the prolonged use of all cephalosporins in the health care setting is actively discouraged in favour of beta-lactam-beta-lactamase inhibitors or carbapenems (A-2 evidence).

\subsubsection{Health care-associated or severe community-acquired} IAIs: There is paucity of data from randomized clinical trials to document the efficacy of various antimicrobial regimens for the treatment of health care-associated or severe community-acquired IAIs. Thus, recommendations for such patients are primarily based on the clinical experience of respected authorities, descriptive studies, or consensus reports from expert committees (Table 7) (B-3 evidence). In general, antimicrobial regimens with a broader spectrum of activity and relatively low rates of resistance are recommended. Monotherapy with piperacillin-tazobactam, imipenem-cilastatin or meropenem are recommended. Ciprofloxacin plus metronidazole can also be used. Combination regimens with third- or fourth-generation cephalosporins (eg, ceftazidime, ceftriaxone or cefepime) plus metronidazole are additional alternatives; however, due to the concern for selection of ESBL- and ampC-producing multiresistant Gram-negative bacilli as discussed above, prolonged and wide-spread use of cephalosporins are generally discouraged (A-2 evidence). For severely ill patients and those with prolonged previous hospitalization or antimicrobial exposure, infection with multiresistant Gram-negative bacilli is more common, and the addition of an aminoglycoside may be desirable to broaden the antimicrobial spectrum and delay the emergence of resistance despite their known toxicities (9) (B-3 evidence).

A subset of moderately ill patients with health care-associated IAIs were studied prospectively in a double-blind, randomized clinical trial involving sequential IV to PO monotherapy with moxifloxacin (102). Moxifloxacin was found to be superior to piperacillin-tazobactam IV $\rightarrow$ amoxicillin-clavulanate PO sequential therapy in this subset analysis (clinical success rates $82 \%$ versus $55 \% ; \mathrm{P}<0.05)$. The bacteriological eradication rates as well as safety profiles in the two study arms were comparable. Moxifloxacin has excellent pharmacokinetic and pharmacodynamic properties, and penetrates well into peritoneal exudates and abscess cavities $(134,135)$. Other potential advantages include once daily dosing, IV to PO switch without dosage adjustment, and convenience as well as cost-savings due 
to shortened hospitalization. If confirmed by additional studies in seriously ill patients, moxifloxacin may become a treatment option for patients with community- as well as health careassociated complicated IAI (B-2 evidence). However, these advantages must be balanced against the potential for fluoroquinolones to select for ESBL-producing pathogens, the emergence of resistance among $B$ fragilis, and the reported association between fluoroquinolone use and superinfection by Clostridium difficile $(89,93)$. Other newer fluoroquinolones with broad-spectrum activity against both Enterobacteriaceae and anaerobes are currently under investigation (71).

Tigecycline has excellent in vitro activity against intraabdominal isolates, including aerobic and anaerobic Grampositive and Gram-negative bacteria, ESBL-producing Enterobacteriaceae, MRSA and vancomycin-resistant enterococci (VRE). It is, however, relatively inactive against $P$ aeruginosa (136). It also has favourable pharmacokinetic and pharmacodynamic properties that theoretically make it a good candidate for the treatment of health care-associated infections if used in combination with ciprofloxacin to cover for $P$ aeruginosa. Unfortunately, in the two phase 3 trials reported, although noninferiority to imipenem-cilastatin was established, the study populations uniformly had only mild to moderate severity of infection (mean APACHE II scores were 6.3 for tigecycline and 6.0 for imipenem-cilastatin). It remains to be seen whether tigecycline is efficacious and safe for seriously ill patients with health care-associated infections.

5.3.3 Empirical anti-MRSA therapy: MRSA is isolated with increasing frequency in both community-acquired and health care-associated infections (137). Patients with prolonged hospitalization, an indwelling intravenous catheter, previous antimicrobial exposure in the preceding three months and a nursing home residence are particularly at risk for MRSA infections (138). However, the role of MRSA in IAIs other than surgical site infections is unclear $(139,140)$. In health care-associated postoperative and surgical site infections, empirical anti-MRSA therapy should be considered for those who are known to be colonized with the organism or have a history of MRSA infection. Vancomycin remains the agent of choice, although quinupristin-dalfopristin, linezolid, daptomycin and tigecycline may also be used. Vancomycin may also be considered for surgical prophylaxis in patients who are known to be MRSA carriers or if they come from facilities with a high prevalence of MRSA infection (141) (B-2 evidence).

5.3.4 Empirical antienterococcal therapy: Enterococci are frequently isolated in intraperitoneal cultures in patients with community-acquired surgical IAIs. However, treatment regimens that provide antienterococcal coverage were not superior to comparative regimens that did not provide such coverage in prospective, blinded and randomized clinical trials $(1,110,142)$. Thus, routine empirical coverage for enterococci for community-acquired, mild to moderately severe surgical IAIs is not required (A-1 evidence). However, enterococci are recovered more commonly in certain populations with complicated IAIs, particularly those with health care-associated, postoperative infections, severe immunosuppression, recurrent infections and those receiving prolonged antimicrobial therapy with cephalosporins that select for enterococci (111). Such individuals are also at increased risk for treatment failure and poor outcome (143). Thus, empirical antienterococcal therapy is recommended for the following at-risk populations until definitive culture results become available: immunosuppressed patients with health care-associated postoperative IAIs; patients with severe sepsis of abdominal origin or recurrent peritonitis who have previously received cephalosporins and other broad-spectrum antimicrobial agents that select for enterococci, patients with biliary sepsis undergoing liver transplantation, and patients with peritonitis and valvular heart disease or prosthetic intravascular devices that place them at high risk for enterococcal endocarditis (C-3 evidence). Initial empirical antienterococcal therapy should be directed at Enterococcus feacalis, which can be treated with penicillin, ampicillin, piperacillin-tazobactam, imipenem-cilastatin, tigecycline or vancomycin (C-3 evidence). Empirical coverage directed against Enterococcus faecium should be considered in patients with prolonged previous antimicrobial exposure, particularly those with hepatobiliary sepsis undergoing liver transplantation, and vancomycin is the agent of choice. Tigecycline or daptomycin may be considered if the isolates are vancomycin resistant (C-3 evidence).

5.3.5 Pre-emptive antifungal therapy: Candida species are not common pathogens in community-acquired IAIs. However, invasive candidiasis is an important cause of mortality in immunosuppressed patients with health care-associated or postoperative IAIs $(144,145)$. Severity of disease (APACHE II of 15 or greater), previous antimicrobial therapy, upper gastrointestinal source of infection, intraoperative cardiopulmonary failure and demonstration of yeast in Gram stain of peritoneal fluids are independent risk factors for culture-documented Candida peritonitis (146). Antifungal therapy should be initiated if Candida species is isolated from intra-abdominal cultures, and fluconazole is the antifungal agent of choice if $\mathrm{C}$ albicans is identified (B-2 evidence). For non-albicans Candida species resistant to fluconazole, an echinocandin (eg, caspofungin, micafungin or anidulafungin) or a triazole (eg, voriconazole) to which the organism is susceptible is appropriate (B-3 evidence). Amphotericin is not recommended for initial antifungal therapy due to its toxicity (B-2 evidence).

Pre-emptive antifungal therapy with either fluconazole or an echinocandin may be considered in immunosuppressed and seriously ill patients who present with recurrent or postoperative IAIs, and are known to be colonized with $\mathrm{C}$ albicans at multiple sites (144) (C-2 evidence). Such a strategy has been shown to prevent the development of invasive candidiasis or candidemia, but has no impact on overall mortality (147-149).

\subsection{Duration of antimicrobial therapy}

The optimal timing and duration of antimicrobial therapy for complicated IAIs have not been determined. Whereas suboptimal antibiotic therapy clearly results in enhanced failure rates and mortality, prolonged antibiotic administration leads to superinfections, selection for resistance, as well as adverse effects and added cost. In various clinical trials evaluating the efficacy and safety of antimicrobial regimens for surgical IAIs, a 'standard' duration of five to 14 days was chosen. However, 
more recent studies have attempted to evaluate whether a shorter course of antimicrobial therapy in community-acquired IAIs might be more cost effective. Schein et al (150) prospectively evaluated the effect of limiting the duration of antimicrobial therapy based on intraoperative findings of the degree of contamination and extent of infection. Treatment for localized peritonitis (eg, perforated appendicitis, cholecystitis, diverticulitis, gastroduodenal or traumatic perforations, strangulated small bowel and colorectal surgery) was restricted to less than $48 \mathrm{~h}$ if adequate source control was achieved. Treatment for more extensive infection, including generalized peritonitis was limited to five days or less. This strategy yielded results comparable with historical controls. Another approach evaluated the effect of limiting the duration of antimicrobial therapy based on the clinical response as assessed by resolution of fever and leukocytosis, and improvement of abdominal examination and gastrointestinal function $(151,152)$. These prospective studies confirmed that similar outcomes could be achieved with earlier cessation of antibiotic treatment based on a satisfactory clinical response. Finally, a prospective, double-blind, multicentre randomized trial was conducted to evaluate the efficacy and safety of administering ertapenem $1 \mathrm{~g}$ per day for three days compared with the 'standard' duration of therapy for five days or more (mean 5.7 days, range five to 10 days) in 111 adults with community-acquired IAIs (116). The clinical and bacteriological response rates in the two groups among 90 evaluable patients were similar $(93 \%$ and $95 \%$, respectively; versus $90 \%$ and $94 \%$ ). It should be noted that disease severity was mild to moderate (mean APACHE II score of 6.2) in all patients, and intraoperative source control was considered adequate in all but one patient. This was the only prospective study that specifically examined the duration of antimicrobial therapy in complicated IAIs by a double-blind randomized study design.

Based on these findings, it is recommended that the duration of antimicrobial therapy for complicated IAIs should be no more than five to seven days, unless it is difficult to achieve adequate source control (B-3 evidence). Treatment duration should be guided by intraoperative findings and clinical response as assessed by resolution of fever and leukocytosis, abdominal examination and gastrointestinal function. Patients who continue to exhibit clinical evidence of infection at the end of seven days should be evaluated for residual infection, resistant microorganisms, noninfectious causes of inflammation and other possible reasons of treatment failure, rather than simply prolonging or broadening antimicrobial therapy (B-3 evidence).

It is further recommended that the duration of antimicrobial therapy for the purpose of surgical prophylaxis should be limited to $24 \mathrm{~h}$ or less in patients with penetrating bowel trauma repaired within $12 \mathrm{~h}$, intraoperative contamination by enteric contents, or nonperforating appendicitis in the absence of abscess or local peritonitis (A-1 evidence).

\subsection{Management of the nonresponsive patient}

Patients with recurrent IAIs following initial surgical and antimicrobial therapy for secondary peritonitis are referred to as having 'tertiary' peritonitis $(6,60)$. These patients are characterized by persistent or worsening organ dysfunction and an inability to localize their peritoneal infection due to poor host defences. Their microbial flora also appears strikingly different, often dominated by $P$ aeruginosa and other nonfermentative Gram-negative bacilli, Enterobacter species, enterococci, Candida species, resistant Bacteroides species and low-virulence organisms such as coagulase-negative staphylococci. These organisms likely represent superinfections from the hospital flora or selection of resistant microbes from previous antimicrobial therapy $(3,153)$. Treatment is difficult because the mortality remains high (exceeding 50\%) despite prolonged systemic antibiotics and aggressive surgical management (63).

In the face of persistent or recurrent IAI, every effort should be made to ensure that adequate source control has been attained. Reimaging by computed tomography or magnetic resonance imaging is required to identify loculated foci of residual infection or anastomotic leak of the intestinal tract. Repeat laparotomy is recommended to achieve adequate source control and to facilitate pathogen-directed antimicrobial therapy by obtaining appropriate samples for microbiological evaluation. Repeat laparotomy may also be indicated for decompression in selected patients with IAH and the ACS (refer to section 7.2). Extra-abdominal sources of infection should be excluded. Every effort should be directed at treating underlying comorbid conditions, mitigating immunosuppression and bolstering innate mucosal immunity. It remains to be determined whether immunotherapy (either humoral or cellular) in conjunction with appropriate antimicrobial therapy will improve clinical outcome in such patients (106).

\subsection{Key recommendations for initial empirical antimicrobial therapy}

Recommendation 9. For patients with community-acquired surgical IAIs with mild to moderate severity (APACHE II score of lower than 15) who have not undergone prolonged previous hospitalization (five days or more) or received previous antimicrobial therapy (more than two days), initial empirical antimicrobial therapy should be directed against 'core pathogens' only, including enteric Gram-positive cocci as well as facultative and anaerobic Gram-negative bacilli, particularly E coli and B fragilis (A-1 evidence). For adult patients, monotherapy with cefoxitin, ticarcillin-clavulanate, ertapenem, moxifloxacin or tigecycline is appropriate; alternatively, combinations of cefuroxime, cefotaxime, ceftriaxone or ciprofloxacin, each with metronidazole, are preferable to broader-spectrum regimens (A-1 evidence).

Recommendation 10. Ampicillin-sulbactam (not available in Canada), cefotetan and clindamycin are no longer recommended for routine empirical therapy of complicated IAIs because of the high rate of resistance among communityacquired E coli against ampicillin-sulbactam, and among $B$ fragilis against cefotetan and clindamycin (B-2 evidence).

Recommendation 11. In light of the availability of less toxic regimens and unfavourable clinical response rates in randomized clinical trials, aminoglycosides are not recommended for routine empirical treatment of complicated IAIs (A-1 evidence).

Recommendation 12. For health care-associated surgical IAIs and seriously ill patients with community-acquired infections (APACHE II score of 15 or greater, previous hospitalization of five days or more, or previous antimicrobial therapy of two days or more), antimicrobial agents with broader spectrum of 
activity against facultative and anaerobic Gram-negative bacilli are recommended (B-2 evidence). For adult patients, monotherapy with piperacillin-tazobactam, imipenem-cilastatin, meropenem, or combinations of ceftazidime, cepefime or ciprofloxacin with metronidazole, or tigecycline in combination with ciprofloxacin are appropriate (B-2 evidence).

Recommendation 13. IV to PO sequential treatment with a fluoroquinolone (such as monotherapy with moxifloxacin or combination therapy with ciprofloxacin plus metronidazole) may be a cost-effective alternative (B-2 evidence). Continued surveillance for emerging resistance, particularly against facultative Gram-negative bacilli, should be implemented and periodic review of their efficacy and safety should be considered when choosing monotherapy or combination therapy with a fluoroquinolone (A-1 evidence).

Recommendation 14. In light of the emerging concern of ESBLproducing Enterobacteriaceae species due to selection pressure by increased use of oxyimino-cephalosporins (ceftazidime, ceftriaxone and cefotaxime), as well as ampC-producing nosocomial pathogens (resistant to all cephalosporins), the prolonged use of all cephalosporins in the health care setting is actively discouraged in favour of beta-lactam-beta-lactamase inhibitors or carbapenems (154,155) (A-2 evidence).

Recommendation 15. Routine coverage for enterococci is not recommended in patients with community-acquired IAI of mild to moderate severity (A-1 evidence). However, empirical antienterococcal therapy should be considered for immunosuppressed patients with health care-associated, postoperative or recurrent IAIs, those with antimicrobial exposure to cephalosporins and other broad-spectrum regimens selecting for enterococci, and those with valvular heart disease or intravascular prosthetic devices (B-3 evidence).

Recommendation 16. Coverage for $P$ aeruginosa should be considered if it is the only pathogen recovered, if it is isolated from blood cultures, or if the patient has not responded to antimicrobial treatment that does not cover $P$ aeruginosa in the setting of health care-associated IAIs (B-2 evidence).

Recommendation 17. Anti-MRSA therapy should be administered for health care-associated IAIs in patients who are known to be colonized with the organism or have a history of MRSA infection. Vancomycin remains the agent of choice, although linezolid, daptomycin, tigecycline and quinupristin-dalfopristin may also be used. Vancomycin may also be considered for surgical prophylaxis in patients who are known to be MRSA carriers or if they come from facilities with a high prevalence of MRSA infection (B-2 evidence).

Recommendation 18. Targeted antifungal therapy is recommended for patients with severe community-acquired or nosocomial IAIs only if Candida species is isolated from intra-abdominal or blood cultures. Fluconazole is the agent of choice if $\mathrm{C}$ albicans is isolated. For non-albicans Candida species, either an echinocandin (such as caspofungin, micafungin or anidulafungin) or a triazole (such as voriconazole) to which the organism is susceptible may be considered (B-2 evidence).

Recommendation 19. Amphotericin B is not recommended as initial therapy because of its toxicity (B-2 evidence).

Recommendation 20. Pre-emptive antifungal therapy with fluconazole or an echinocandin may be considered for seriously ill patients with high risk for invasive candidiasis (eg, immunosuppression, postoperative or recurrent peritonitis, Candida colonization at multiple sites, etc); however, such a strategy has not been shown to impact mortality (C-2 evidence).

Recommendation 21. The duration of antimicrobial therapy should be guided by intraoperative findings and clinical response as assessed by resolution of fever and leukocytosis, abdominal examination and gastrointestinal function, and should be no more than five to seven days unless it is difficult to achieve adequate source control (B-3 evidence).

Recommendation 22. Patients who continue to exhibit clinical evidence of infection at the end of seven days of antimicrobial therapy should be evaluated for residual infection, resistant microorganisms and other possible causes of treatment failure rather than simply prolonging or broadening antimicrobial therapy (C-3 evidence).

Recommendation 23. In patients with postoperative or other health care-associated infections and those with clinical treatment failure, the acquisition or selection of resistant microorganisms should be strongly suspected, and further antimicrobial therapy should be guided by intraoperative cultures and susceptibility testing obtained directly from abscess fluid or the peritoneal cavity (B-2 evidence).

Recommendation 24. Development of clinical pathways (ie, a protocol approach) for the management of complicated IAIs based on local epidemiology of antimicrobial utilization and antibiotic resistance profiles is highly recommended. Such locally developed clinical pathways should standardize the approach to diagnosis, microbiological and radiological investigations, empirical antimicrobial therapy as well as policies regarding discharge and outpatient management. Such local guidelines should be established by a multidisciplinary team including surgeons, infectious disease and medical microbiology specialists, emergency physicians and other health care providers, and should reflect local resources and local standards of care (B-3 evidence).

Recommendation 25. Each institution should develop its own suite of performance measures to provide ongoing evaluation of the effectiveness and appropriateness of the local clinical pathways for complicated IAIs, ultimately leading to improved quality of care (B-3 evidence).

\section{SOURCE CONTROL}

Complicated IAIs typically originate from a leak in the gastrointestinal tract and cannot be eradicated without first addressing the original source of bacterial contamination. The term 'source control' is derived from the environmental health literature and refers to efforts to reduce the amount of materials entering the waste stream from a particular source. Specifically, it refers to actions that prevent pollution at its origin (United States Environmental Protection Agency's Handbook of Groundwater Protection and Cleanup Policies for RCRA Corrective Action <www.epa.gov/correctiveaction>). Similarly, source control of IAIs in this context refers to any intervention directed toward the primary origin of the infectious process. There is little debate regarding the primacy of source control in the management of IAIs. Ideal antimicrobial therapy cannot mitigate the effects of poor or absent source control. In retrospective cohort studies, the strongest risk factor for recurrent infection (or failure) is poor source control, attesting to the critical importance of appropriate surgical interventions (A-2 evidence) $(47,156)$. 


\subsection{Approaches to source control}

Source control of complicated IAIs can be achieved using either operative or percutaneous approaches. The ideal approach depends on specific patient and local factors, including the source and extent of infection, and available resources. While there are some grey areas that are evolving (see below), in most cases, the approach to assure adequate source control is not controversial (157).

Operative source control is required when adequate source control cannot be achieved percutaneously. Typically, these circumstances are characterized by a need to resect a gangrenous or perforated viscus, defunction (divert the enteric stream) a more distal perforation of the gastrointestinal tract with an ileostomy or colostomy, or patch a perforated ulcer (A-3 evidence). Diffuse IAI or abscesses that cannot be accessed percutaneously also need to be drained using an operative approach (A-3 evidence). While conventional wisdom suggests that all abscesses need some form of drainage, there is increasing evidence suggesting that the relatively small (less than $3 \mathrm{~cm}$ ) abscesses might not require drainage (B-2 evidence). These data are drawn primarily from studies of diverticular and renal abscesses, and might not necessarily be applicable to other clinical settings $(158,159)$.

In the case of multiple intra-abdominal abscesses or very complex abscesses, either approach is acceptable. Complex abscesses are those with multiple loculations or those that have significant debris or whose contents are viscous (eg, pancreatic or fungal abscesses) and have a higher probability of failure using percutaneous approaches (160). However, if these can be approached percutaneously and the patient has sufficient physiological reserve to tolerate multiple drains and several attempts at source control, then this approach may be acceptable (B-3 evidence). In select cases, the use of thrombolytic agents administered through the drains might facilitate drainage and increase the probability of success (C-2 evidence) $(161,162)$. If the patient is critically ill, has limited physiologic reserve or is suffering from overt ACS, it might be more expedient to simply address the source of infection using an operative approach (B-3 evidence).

There are two clinical settings in which accruing evidence is challenging the need for source control. Perforated diverticulitis is typically managed through resection of the sigmoid colon followed by either anastomosis or colostomy. In this context, definitive source control is achieved by resecting the colon with its perforation. However, there are several reports suggesting that laparoscopic irrigation of the peritoneal cavity and drainage of the perforation is sufficient and reduces the need for emergency resection with or without colostomy (163-166). Many of these reports have included patients with fecal peritonitis, with reasonable rates of success. Because none of these studies were randomized, the recommendation supporting this approach is relatively weak (C-2 evidence).

The approach to infected pancreatic necrosis is also evolving. Source control in this context traditionally mandates operative necrosectomy. However, data from multiple case series suggest that in select patients, percutaneous approaches might be acceptable (B-2 evidence) (167-171). Over time, infected necrosis typically undergoes liquefaction, ultimately forming a pancreatic abscess. In a critically ill and deteriorating patient with evidence of infected pancreatic necrosis that is relatively early in its course, the limited degree of liquefaction mandates debridement as the operative intervention and such a patient might not be a suitable candidate for percutaneous drainage. By contrast, later in the course, liquefaction might have progressed sufficiently to allow for percutaneous management. Thus, the choice of technique depends on the anatomical position, the ratio of solid to fluid components within the collection and the degree of systemic organ dysfunction (169). If the patient has sufficient physiological reserve and can wait for further liquefaction to occur to allow for percutaneous drainage, then this might be the preferred approach.

\subsection{Key recommendations for source control}

Recommendation 26. Adequate source control is the primary means of managing IAI and should not be subjugated to antimicrobial therapy (A-2 evidence).

Recommendation 27. Operative approaches to source control should be used when it is necessary to resect a gangrenous or perforated viscus, patch a perforated viscus, divert the enteric stream, decompress overt ACS, or when percutaneous approaches to abscess drainage are not possible or have not been effective (A-3 evidence).

Recommendation 28. Small abscesses (less than $3 \mathrm{~cm}$ ) might be amenable to antimicrobial therapy without drainage (B-2 evidence).

Recommendation 29. In select patients, source control of perforated diverticulitis can be achieved using laparoscopic lavage and drainage (C-2 evidence).

Recommendation 30. In select patients, source control of infected pancreatic necrosis can be achieved using percutaneous approaches (B-2 evidence).

\section{IAH AND ACS}

\subsection{Definitions and pathophysiology}

Pathologically raised IAP can be caused by a variety of conditions including complicated IAIs and the ACS. Sustained elevations in IAP have severe pathophysiological effects including impaired venous return and elevated cardiac afterload, increased difficulty in ventilation due to restricted lung volumes and atelectasis, impaired perfusion of the viscera including the kidneys, liver and the gastrointestinal tract, ultimately leading to multiple organ failure (172-176). Moderate increases in IAP have been shown to cause bacterial translocation from the gastrointestinal tract in animal models $(177,178)$. ACS is a potentially lethal complication caused by any event that produces sustained IAH. Precipitating events include acute pancreatitis, ruptured abdominal aortic aneurysm, blunt abdominal trauma with intra-abdominal bleeding from splenic, hepatic and mesenteric injuries, and complicated IAI. Risk factors that predispose to either IAH or ACS are shown in Table 8. The World Society on the Abdominal Compartment Syndrome (WSACS) developed a consensus statement in 2006 that defined IAH as maximum or sustained IAP at or above $12 \mathrm{mmHg}$ (179). ACS is defined as IAP above $20 \mathrm{mmHg}$ with evidence of new organ dysfunction or failure. In addition to IAP, another potentially useful measurement is the abdominal perfusion pressure (APP) (179), which can be derived from the mean arterial pressure (MAP) using the formula: APP = MAP - IAP. This is an easily measured parameter and may be superior to IAP for monitoring critically ill patients. In a retrospective study of 
144 critically ill surgical patients with intermittent IAP measurements, Cheatham et al (180) noted that an APP threshold of $50 \mathrm{mmHg}$ proved superior to either MAP or IAP alone in predicting patient survival from IAH and ACS.

It should be recognized that IAH is neither infrequent, nor insignificant. Malbrain et al $(181,182)$ reported a high occurrence of IAH (greater than $12 \mathrm{mmHg}$ ) including one-half of all patients in a multi-institutional point-prevalence study, as well as $32 \%$ of a second prospective evaluation of critically ill patients within 13 intensive care units in six countries. The presence of sustained IAH or ACS is associated with a significant increase in mortality (183). Bodnar et al (184) recently reported that $66 \%$ of general surgery patients developed IAH and $13 \%$ developed ACS. Mortality rates were $16 \%$ and $42 \%$ in those with IAH and ACS respectively, significantly higher than the mortality rate of $9 \%$ among those without IAH. A recent study from Canada also revealed a significant relationship between IAH and death before intensive care unit discharge, as well as organ dysfunction and requirement for renal replacement therapy (PB McBeth and AW Kirkpatrick, unpublished data). Despite their grave consequences, both IAH and ACS are poorly recognized in the routine practice of critical care in Canada.

\subsection{IAH and complicated IAI}

An association between IAH and increased rates of organ failure and death in cases of severe pancreatitis has been repeatedly demonstrated (185-187). Leppaniemi and Kemppainen (188) suggested that many of the early deaths in cases of necrotizing pancreatitis, previously believed to be due to overwhelming systemic inflammatory response syndrome, were actually due to undiagnosed and untreated ACS. Plantefeve et al (189) reviewed the recent literature regarding any association of IAH or ACS with intra-abdominal sepsis and concluded that they were closely interrelated. Malbrain et al (182) also reported the presence of IAH in $80 \%$ of patients with abdominal infections in a prospective, multinational, point prevalence study in critically ill patients. Busani et al (190) evaluated the relationship between IAP and clinical outcome in 22 patients with urgent abdominal surgery for a variety of intra-abdominal processes including 12 with IAIs or other inflammatory conditions. Mortality among those with IAH was $28 \%$ versus $12 \%$ in patients without IAH. It should be noted that although the presence of IAH is clearly associated with increased morbidity and mortality in critically ill patients, a direct causal relationship between IAH and adverse outcome has not yet been established. To confirm a causal relationship, it would be critical to demonstrate that effective treatment of IAH or ACS leads to reduced mortality, but such data currently do not exist. Further research and controlled trials are urgently required, recognizing that patients with complicated IAIs have multiple predisposing risk factors for either IAH or ACS.

\subsection{Management of IAH and ACS in complicated IAIs}

Few prospective, randomized treatment data exist in the published literature for the management of patients with complicated IAIs and IAH. Although IAH in the setting of pancreatitis has been treated with a variety of modalities including open abdominal laparotomies (191-193), continuous renal replacement therapy (194) and a novel modified subcutaneous
TABLE 8

Risk factors associated with intra-abdominal hypertension or abdominal compartment syndrome

Diminished abdominal wall compliance

Acute respiratory failure, especially with elevated intrathoracic pressure

Abdominal surgery with primary fascial closure

Major trauma or burns

Prone positioning

Increased intraluminal contents

Gastroparesis

lleus

Colonic pseudo-obstruction

Increased abdominal contents

Hemoperitoneum or pneumoperitoneum

Ascites or liver dysfunction

Capillary leak or fluid resuscitation

Acidosis ( $\mathrm{pH}<7.2)$

Hypotension

Hypothermia (core temperature $<33^{\circ} \mathrm{C}$ )

Multiple transfusions ( $>10$ units of blood over $24 \mathrm{~h}$ )

Coagulopathy (platelets $<55,000 / \mathrm{mm}^{3}$, or activated partial

thromboplastin time $>2$ times normal, or prothrombin time $<50 \%$, or

international normalized ratio $>1.5$ )

Massive fluid resuscitation ( $>5 \mathrm{~L}$ over $24 \mathrm{~h}$ )

Oliguria

Sepsis

Major trauma or burns

Damage control laparotomy

Adapted from reference 176

fasciotomy (195), there are no definitive data that any of these therapies improves patient outcomes. The WSACS recently published consensus guidelines regarding the management of IAH and ACS in the care of the critically ill $(196,197)$. The following are recommended:

1. A baseline IAP measurement should be obtained in all critically ill patients if two or more risk factors for IAH or ACS are present (Table 8) (B-2 evidence);

2. If IAH (greater than $12 \mathrm{mmHg}$ ) is present, serial IAP measurements should be performed throughout the patient's critical illness (A-3 evidence); and

3. The management of IAH or ACS is based on four general principles: serial monitoring of IAP, optimization of systemic perfusion and organ function in the face of IAH, institution of specific medical procedures to reduce IAP and its effects on end-organ function, and prompt surgical decompression of refractory IAH (B-1 evidence).

Given the high mortality rate and grave consequences of IAH in complicated IAI, the WSACS recommendations to monitor IAP more regularly in critically ill patients seem reasonable, particularly because it is a relatively simple and lowcost procedure that typically requires only a bladder catheter connected to a pressure transducer monitor. In addition to the current standard of intermittent bladder pressure measurements, gastric tonometry has also been found to correlate well with more invasive direct pressure measurements from the peritoneal cavity (198). The specific measures to reduce IAP and improve multiorgan function in critically ill patients are beyond the scope of this guideline, but can be found on the WSACS Web site < www.wsacs.org>. 


\subsection{Key recommendations for IAH In IAIs}

Recommendation 31. The presence of risk factors that may predispose to the development of IAH or ACS should be assessed in all acutely ill patients (APACHE II score greater than 15) with complicated IAI (B-2 evidence).

Recommendation 32. Baseline IAP measurements should be determined in all critically ill patients with complicated IAIs if two or more risk factors for IAH or ACS are present (B-2 evidence). If IAH (IAP greater than $12 \mathrm{mmHg}$ ) or ACP (IAP greater than $20 \mathrm{mmHg}$ ) is present, serial IAP measurements should be performed throughout the patient's critical illness (A-3 evidence).

Recommendation 33. Surgical decompression should be considered for patients with refractory IAH or evidence of ACS. In addition, medical approaches to reduce IAP and associated endorgan dysfunction should be implemented, consistent with local practices in the care of critically ill patients (B-2 evidence).

\section{INFECTION CONTROL AND PREVENTION}

Infection control issues in IAIs can be divided into three broad categories: a) patient-related risk factors for postoperative and surgical site infections; b) prevention of nosocomial infections by improving the operating room environment, use of topical antiseptics and antimicrobial prophylaxis for surgical site infections; and c) preventing the spread of antibiotic-resistant organisms (ARO) through surveillance and antibiotic stewardship. The incidence of surgical site infections increases dramatically in the presence of intestinal spillage and gross peritoneal contamination. Administration of preoperative systemic antibiotics, use of wound protective devices and lavage of surgical wounds at the end of operative procedures do not reliably prevent this complication. Meticulous postoperative care in wound dressing, intravenous catheter maintenance, skin antisepsis, and close attention to principles of infection control are the cornerstones to successful management (199). Preventing the spread of multiresistant pathogens in this patient population (especially ESBL-producing Enterobacteriaceae species, $\mathrm{C}$ difficile, MRSA and VRE) is a high priority.

It is important to recognize that the application of individual infection control measures is unlikely to have a major impact on outcome. Rather, it is the application of all the different intervention strategies that will decrease the risk of postoperative and surgical site infections following abdominal surgery. A hospital-wide surgical site infection surveillance program with continuous collaboration and feedback to surgeons has been proven to reduce the incidence of surgical site infections $(200,201)$ (A-1 evidence). The National Nosocomial Infection Surveillance (NNIS) risk index with a score ranging from 0 to 3 is a valid tool for monitoring surgical site infections in a surveillance program $(27,202)$.

\subsection{Patient-related issues}

Certain risk factors such as diabetes, obesity, smoking and malnutrition have been shown to play a role in increasing surgical site infections (203). Avoiding hyperglycemia perioperatively and encouraging tobacco cessation at least 30 days before elective surgery are well-recognized preventive measures $(204,205)$ It is advisable to instruct the patient to shower with an antiseptic agent the night before the surgical procedure (B-2 evidence) (200). Products containing chlorhexidine gluconate have the biggest impact on bacterial burden reduction. Hair removal is indicated only in cases in which hair may hamper the surgical procedure itself. If hair has to be removed, it should be performed immediately before surgery using electric clippers (A-1 evidence) (200). Hair removal more than $24 \mathrm{~h}$ before surgery is not advised (A-1 evidence).

Three types of topical antiseptic agents are currently used for skin preparation of the operative site and for preoperative scrubbing by the surgical team, including alcohol-based agents, iodophors (providone-iodine) and chlorhexidine gluconate (Appendix 4). No data have ever shown superiority of one agent over another. Combination agents such as chlorhexidine and alcohol together have been shown to have better residual antimicrobial activity than single agents alone (200).

\subsection{The operating room and related issues}

The operating room environment should meet certain standards. A minimum of 15 air changes per hour with at least three air changes of outdoor fresh air is required. Physical parameters should meet the following criteria: a room temperature between $20^{\circ} \mathrm{C}$ to $23^{\circ} \mathrm{C}$, and a relative humidity of $30 \%$ to $60 \%$. The operating room must also be maintained at positive pressure with regard to the corridors and outside areas. A two-filter system installed in series should also be in place, and air should enter the room through the ceiling and exhaust near the floor (B-2 evidence) $(206,207)$. A laminar airflow system is not warranted for abdominal surgeries. The use of this system has mainly been studied for orthopedic procedures, and no data supporting its use in abdominal surgery exist (200). An annual check of the physical parameters of the operating room and a complete maintenance program should be instituted at the local level (B-3 evidence).

The surgical team should adopt an approved scrubbing procedure for at least $2 \mathrm{~min}$, which includes the hands, arms and elbows (A-2 evidence) (200). Numerous alcohol-chlorhexidine antiseptic solutions are commercially available and adequate as topical antiseptic agents (208). Artificial nails should not be worn; nail polish should not be scaled and jewellery should be disallowed. Masks are mainly used as a protective gear against splashing of potentially infectious body fluids. The occurrence of an unusually high number of postoperative infections involving S aureus or group A streptococcus should prompt an investigation for a common source reservoir among the operating room personnel. Routine screening for these pathogens is not indicated and should not be performed.

\subsection{Antimicrobial prophylaxis for surgical site infections}

Surgical antimicrobial prophylaxis plays a key role in abdominal surgery (209). The main objective is not to sterilize the surgical field, but to decrease the bacterial burden as much as possible at a critical time. Intravenous bactericidal antibiotics with an in vitro spectrum that covers the most likely intraoperative microorganisms should be administered in a timely fashion $(199,210)$. In intra-abdominal surgical procedures, $S$ aureus, Gram-negative bacilli and anaerobes from the distal part of the digestive tract are the main target pathogens. The vast majority of abdominal surgeries for which antimicrobial prophylaxis is required are classified as clean-contaminated. The abdominal procedures for which antimicrobial prophylaxis is indicated are listed in Table 9. Recommended prophylactic 
antibiotic regimens are shown in Table 10. The following principles are the cornerstone of surgical antimicrobial prophylaxis:

1. Antibiotics should be used only if evidence of benefit is available from clinical trials, and in situations for which a postoperative surgical site infection would have major consequences (A-1 evidence) (200).

2. Timing of antibiotic infusion is critical so that the peak of tissue concentrations is obtained at the moment of incision, typically $30 \mathrm{~min}$ to $60 \mathrm{~min}$ before the time of incision (A-1 evidence) (210).

3. Therapeutic serum and tissue levels should be maintained throughout surgery and ideally a few hours after completion of the procedure. In cases of prolonged surgical procedures, prophylactic antibiotics may need to be readministered intraoperatively (B-2 evidence) (200).

4. The total duration of antimicrobial prophylaxis for abdominal surgery should not exceed $24 \mathrm{~h}$.

\subsection{Prevention of the spread of antibiotic-resistant microorganisms}

MRSA is a growing concern among Canadian institutions (211). Rates have been multiplied by a factor of 10 within the past decade. Compared with methicillin-sensitive $S$ aureus, MRSA infections have been associated with increased mortality and morbidity. In addition, Canadian data have shown that the average increased cost per MRSA infection is more than $\$ 14,000$. Available antibiotics are either relatively ineffective for severe infections (vancomycin) or extremely costly (eg, linezolid and daptomycin). Increasing minimum inhibitory concentration of vancomycin $(1 \mathrm{mg} / \mathrm{L}$ or greater) among MRSA strains is associated with an increased risk of treatment failure and is a serious concern (212).

Infections due to VRE remain rare in Canada and tend to occur in clusters. When found, they can involve the peritoneal cavity and, even more commonly, the biliary tract. Linezolid remains the best available agent against invasive infections caused by this microorganism.

To control the spread of AROs, an effective infection control program must be implemented in all institutions (A-1 evidence) (213). This should include a comprehensive hand hygiene program that has been proven to decrease the overall incidence of MRSA and VRE (214). Contact precautions, including gowns and gloves as well as patient and staff cohorting, have been advocated as methods of limiting the transmission of AROs.

\subsection{Key recommendations for infection control and prevention}

Recommendation 34. General measures important for reducing the risk of surgical site infections, such as avoiding hyperglycemia perioperatively, cessation of tobacco use at least 30 days before elective surgery, instructing the patient to shower with an antiseptic agent the night before the surgical procedure, etc, should be instituted in all patients undergoing intra-abdominal surgery (B-2 evidence). Hair removal is indicated only in cases where the hair may hamper the surgical procedure itself. If hair has to be removed, it should be performed immediately before the surgery using electric clippers (A-1 evidence).

\section{TABLE 9}

Clean-contaminated abdominal surgeries for which antibiotic prophylaxis is indicated

\begin{tabular}{l} 
Colorectal surgery \\
Elective colon resection, low-anterior resection of the rectum and abdominal \\
perineal resection of the rectum also require a specific colon preparation to \\
empty the bowel contents and reduce the amount of microorganisms \\
Appendectomy \\
Perforated, gangrenous or necrotizing appendicitis \\
Esophageal surgery \\
If obstruction is present \\
Sclerotherapy of esophageal varices \\
Esophageal dilation \\
High-risk gastroduodenal surgery \\
Situations in which gastric acidity is decreased \\
Cancer, active bleeding \\
Decreased gastroduodenal motility (obstruction, obesity) \\
Small-intestine surgery \\
\hline High-risk biliary tract surgery \\
Age $>70$ years, diabetes, obesity \\
Acute cholecystitis, cholelithiasis or obstructive jaundice \\
Nonfunctional gallbladder (excluding nonurgent laparoscopic \\
Open biliary tract surgery
\end{tabular}

Recommendation 35. Surgical team members should adopt a recommended scrubbing procedure for at least $2 \mathrm{~min}$, including hands, arms and elbows (A-2 evidence).

Recommendation 36. A two-filter system installed in series should be in place in the operating room to ensure a clean environment, and air should enter the operating room through the ceiling and exhaust near the floor (B-2 evidence). Regular check-up of all physical parameters of the operating room and a complete maintenance program should be instituted at the local level (B-3 evidence).

Recommendation 37. Antibiotics for surgical prophylaxis should be used only if evidence from clinical trials is available, and in situations for which a surgical site infection may have major consequences (A-1 evidence). If surgical prophylaxis is to be administered, both timing and dosing of the antibiotic infusion should be adjusted to attain peak tissue concentrations at the moment of incision and throughout surgery (A-1 evidence). In cases of prolonged surgical procedures, prophylactic antibiotics may need to be readministered intraoperatively (B-2 evidence).

Recommendation 38. The duration of antimicrobial therapy for the purpose of surgical prophylaxis in the absence of established infection should be limited to $24 \mathrm{~h}$ or less in patients with penetrating bowel trauma repaired within $12 \mathrm{~h}$, intraoperative contamination by enteric contents, or nonperforating appendicitis in the absence of abscess or local peritonitis (A-1 evidence).

Recommendation 39. A hospital-wide surgical site infection surveillance program with continuous collaboration and feedback with the surgical team should be implemented to reduce surgical site infections (A-1 evidence).

Recommendation 40. To effectively control the spread of antibiotic-resistant organisms, an effective infection control program coupled with a rigorous antibiotic stewardship program should be implemented locally (A-1 evidence). 
TABLE 10

Antimicrobial regimens for prophylaxis in abdominal surgery

\begin{tabular}{|c|c|c|c|c|}
\hline Surgery & Likely microorganism & First choice & Second choice & Comments \\
\hline $\begin{array}{l}\text { Esophageal, gastroduodenal } \\
\text { or biliary tract }\end{array}$ & GNB, GPC & Cefazolin 1-2 g IV & $\begin{array}{l}\text { Clindamycin } 900 \text { mg + } \\
\text { gentamicin (2 mg/kg IV) }\end{array}$ & $\begin{array}{l}\text { Vancomycin if MRSA } \\
\text { suspected }\end{array}$ \\
\hline Colorectal & $\begin{array}{l}\text { GNB, Enterococcus, } \\
\text { anaerobes }\end{array}$ & $\begin{array}{l}\text { Cefazolin 1-2 g IV + metronidazole } \\
500 \text { mg IV or cefoxitin } 2 \text { g IV }\end{array}$ & $\begin{array}{l}\text { Clindamycin } 900 \text { mg + } \\
\text { gentamicin }(2 \mathrm{mg} / \mathrm{kg} \text { IV) }\end{array}$ & $\begin{array}{l}\text { Ciprofloxacin } 400 \text { mg + } \\
\text { metronidazole may be an } \\
\text { alternative in selected patients }\end{array}$ \\
\hline Appendectomy & GNB & $\begin{array}{l}\text { Cefazolin 1-2g IV + metronidazole } \\
500 \text { mg IV or cefoxitin 1-2 g IV }\end{array}$ & $\begin{array}{l}\text { Clindamycin or metronidazole + } \\
\text { gentamicin (2 mg/kg IV) }\end{array}$ & \\
\hline
\end{tabular}

GNB Gram-negative bacilli; GPC Gram-positive cocci; IV Intravenous; MRSA Methicillin-resistant Staphylococcus aureus

\section{UNANSWERED QUESTIONS AND FUTURE DIRECTIONS}

Many controversies in the management of complicated IAI remain. More prospective, randomized clinical trials are needed in patients at high risk for treatment failure or mortality. More accurate methods for risk assessment and stratification are needed to aid in the selection of initial antimicrobial therapy. The optimal timing and duration of antimicrobial therapy remains to be determined.

Several newer broad-spectrum antimicrobials are either investigational or recently marketed in North America, and further clinical studies are needed to determine their role in the treatment of complicated IAI. Ceftobiprole, which has recently been marketed in Canada, has broad-spectrum in vitro activity against Gram-positive cocci, including MRSA and methicillin-resistant Staphylococcus epidermidis, penicillin-resistant Streptococcus pneumoniae, E faecalis, facultative Gramnegative bacilli (including ampC-producing $E$ coli and $P$ aeruginosa, but not ESBL-producing strains) (215). Like other third-and fourth-generation cephalosporins, ceftobiprole demonstrates limited activity against $B$ fragilis and non-fragilis Bacteroides species (215). Due to its lack of activity against the predominant colonic anaerobes, this agent would have to be used in combination with an antianaerobic agent such as metronidazole. Doripenem, an investigational carbapenem in Canada but already available in the United States, possesses broad-spectrum in vitro activity against many Gram-positive, Gram-negative and anaerobic bacteria. Like other carbapenems (eg, meropenem), doripenem lacks activity against $E$ faecium, MRSA and Stenotrophomonas maltophilia (216). Doripenem has been studied in complicated IAIs and found to be noninferior in terms of bacteriological and clinical efficacy as well as safety (216). Further clinical trials are required to establish its exact role in the treatment of complicated IAIs.

Although the importance of surgical source control in complicated IAIs is well recognized, it is surprising that only one prospective study (101) has evaluated the adequacy of surgical interventions in addition to antimicrobial therapy. The role of enterococci, coagulase-negative staphylococci, MRSA and Candida species in complicated IAI must be assessed on an individual basis. The emerging resistance among Enterobacteriaceae species, $\mathrm{C}$ difficile and B fragilis will continue to be an issue even as new antimicrobial agents are developed to overcome them. Finally, the ability to treat or eliminate comorbid conditions, mitigate immunosuppression and bolster host defences will be the ultimate challenge.
ACKNOWLEDGEMENTS: The working group thanks Dr Nicolas Christou and Dr Jeff Fuller for developing search terms and assisting with the literature review, and acknowledges the administrative assistance of Core Health Services Inc (Toronto, Ontario) for the preparation of these guidelines. Core Health Services Inc declares no conflict of interest. The working group also thanks Dr Ori Rotstein and Dr Joseph Solomkin for their assistance and review of the guidelines.

FINANCIAL SUPPORT: Financial assistance for the development of these guidelines was provided in the form of arm's-length grants-in-aid from AstraZeneca Canada Inc, Bayer Canada Inc, Merck Frosst Canada Ltd and Wyeth Pharmaceuticals Canada Inc. Sponsors were not involved in any aspect of the guidelines development, the literature interpretation, the decision to publish or any other aspect of the guidelines. The funds were used for conference calls and for organization of and travel to meetings for the members of the Expert Author Group. None of the members of the author group received any financial or in-kind remuneration for their contribution to this work.

POTENTIAL CONFLICT OF INTEREST DECLARED BY WORKING GROUP MEMBERS: AWC owns stock options from Inimex Pharmaceuticals Inc and Migenix Pharmaceuticals Inc, and has received paid consultancy, speaker fees and travel assistance from Merck Frosst Canada. He is also a panel member of the Practice Guidelines for the Diagnosis and Management of Complicated Intra-abdominal infections in Adults and Children by the Surgical Infections Society and the Infectious Diseases Society of America. He is also a member of the Expert Advisory Board on Anti-infective Therapies of Health Canada, and a member of the Canadian Institutes of Health Research UniversityIndustry Grants Peer Review Committee. ABN received speaker fees from Wyeth Pharmaceuticals Inc, and is also a panel member of the Practice Guidelines for the Diagnosis and Management of Complicated Intra-abdominal infections in Adults and Children by the Surgical Infections Society and the Infectious Diseases Society of America. AWK received an unrestricted educational grant from Urodynamix Corp for an unrelated original research project investigating intra-abdominal pressure in weightlessness. KW received ongoing consultancy from Abbott, Pfizer, Roche and GlaxoSmithKline and speaker fees from Abbott, Pfizer, GlaxoSmithKline and Bayer. In addition, he has received research grants from Optimer Pharmaceuticals Inc, and Theravance Pharmaceuticals Inc. GGZ received research grants from Astellas, Abbott, Bayer, Cangene, Merck Frosst, Janssen Ortho, Pfizer, TaiGen, Targanta and Wyeth Pharmaceuticals Inc. GAE, CGB, $\mathrm{GH}$, and GKMH have no conflicts. 
APPENDIX 1

In vitro antimicrobial activity against facultative Gram-positive cocci associated with intra-abdominal infections

\begin{tabular}{|c|c|c|c|c|c|c|c|c|c|c|c|}
\hline Bacteria & $\begin{array}{l}\text { Ceftazidimel } \\
\text { cefepime }\end{array}$ & $\begin{array}{l}\text { Ceftriaxonel } \\
\text { cefotaxime }\end{array}$ & $\begin{array}{c}\text { Fluoro- } \\
\text { quinolones* }\end{array}$ & Clindamycin & $\begin{array}{c}\text { Amino- } \\
\text { glycosides }\end{array}$ & Ertapenem & $\begin{array}{l}\text { Imipenem/ } \\
\text { meropenem }\end{array}$ & $\begin{array}{l}\text { Pip-tazol } \\
\text { ticar-clav }\end{array}$ & Linezolid & Vancomycin & $\begin{array}{l}\text { Tige- } \\
\text { cycline }\end{array}$ \\
\hline $\begin{array}{l}\text { Staphylococcus } \\
\text { aureus (MS) }\end{array}$ & + & $++/+++$ & +++ & ++++ & ++ & ++++ & ++++ & ++++ & ++++ & ++++ & ++++ \\
\hline $\begin{array}{l}\text { Staphylococcus } \\
\text { epidermidis } \\
\text { (MS) }\end{array}$ & + & $++/+++$ & +++ & +++ & + & ++ & ++++ & ++++ & ++++ & ++++ & ++++ \\
\hline $\begin{array}{l}\text { Enterococcus } \\
\text { faecalis (VS) }\end{array}$ & - & - & + & ++ & + & - & +++ & ++++ & ++++ & ++++ & ++++ \\
\hline E faecalis (VR) & - & - & - & $-1+$ & + & - & + & + & ++++ & - & ++++ \\
\hline $\begin{array}{l}\text { Enterococcus } \\
\text { faecium (VS) }\end{array}$ & - & - & + & $-1+$ & + & - & + & + & ++++ & & ++++ \\
\hline
\end{tabular}

*Includes ciprofloxacin, levofloxacin and moxifloxacin; ${ }^{\dagger}$ Includes gentamicin, netilmicin, tobramycin and amikacin. - Poor activity; + Limited activity and/or resistance $\geq 15 \%$; ++ Moderate to good activity and/or resistance $10 \%$ to $14 \%$; +++ Very good activity and/or resistance $5 \%$ to $9 \%$; ++++ Excellent activity and/or resistance <5\%; MR Methicillin resistant; MS Methicillin sensitive; Pip-tazo Pipercillin-tazobactam; Ticar-clav Ticarcillin-clavulanate; VR Vancomycin resistant; VS Vancomycin sensitive. Data adapted from references 216,248-255

\section{APPENDIX 2}

In vitro antimicrobial activity against facultative and aerobic Gram-negative bacilli associated with intra-abdominal infections

\begin{tabular}{|c|c|c|c|c|c|c|c|c|}
\hline Bacteria & $\begin{array}{c}\text { Ceftazidimel } \\
\text { cefepime* }^{*}\end{array}$ & $\begin{array}{l}\text { Ceftriaxonel } \\
\text { cefotaxime }\end{array}$ & $\begin{array}{c}\text { Fluoro- } \\
\text { quinolones }^{\dagger}\end{array}$ & $\begin{array}{c}\text { Amino- } \\
\text { glycosides }^{\ddagger}\end{array}$ & $\begin{array}{c}\text { Erta- } \\
\text { penem }\end{array}$ & $\begin{array}{l}\text { Imipenem/ } \\
\text { meropenem }\end{array}$ & $\begin{array}{l}\text { Pip-tazol } \\
\text { ticar-clav }\end{array}$ & $\begin{array}{c}\text { Tige- } \\
\text { cycline }\end{array}$ \\
\hline Acinetobacter species & ++ & $+/++$ & +++ & +++ & - & +++ & +++ & +++ \\
\hline Citrobacter freundii & $++/+++$ & $++/+++$ & ++++ & ++++ & ++++ & ++++ & $+++/++++$ & NA \\
\hline Enterobacter aerogenes & $++/+++$ & $++/+++$ & ++++ & ++++ & ++++ & ++++ & $+++/++++$ & +++ \\
\hline Enterobacter cloacae & $++/+++$ & $++/+++$ & ++++ & $++/+++$ & ++++ & ++++ & ++ & +++ \\
\hline Escherichia coli & ++++ & ++++ & +++ & ++++ & ++++ & ++++ & ++++ & ++++ \\
\hline E coli (ESBL) & - & - & ++ & +++ & ++++ & ++++ & $-1+$ & +++ \\
\hline Klebsiella pneumoniae & ++++ & ++++ & ++++ & ++++ & ++++ & ++++ & ++++ & ++++ \\
\hline K pneumoniae (ESBL) & - & - & +++ & ++++ & ++++ & ++++ & $-1+$ & +++ \\
\hline K pneumoniae (ampC) & ++ & ++ & ++++ & ++++ & +++ & ++++ & $-1+$ & ++++ \\
\hline Morganella morganii & $++/+++$ & $++/+++$ & ++++ & $+++/++++$ & ++++ & ++++ & ++++ & NA \\
\hline Proteus mirabilis & ++++ & ++++ & $+++/++++$ & ++++ & ++++ & ++++ & ++++ & + \\
\hline Proteus vulgaris & ++ & ++ & ++++ & $+++/++++$ & ++++ & ++++ & ++++ & NA \\
\hline Providencia rettgeri & $++/+++$ & +++ & +++ & + & +++ & ++++ & ++++ & NA \\
\hline Providencia stuartii & $++/+++$ & +++ & +++ & ++ & +++ & ++++ & ++++ & NA \\
\hline Pseudomonas aeruginosa & +++ & + & +++ & +++ & - & +++ & +++ & $-1+$ \\
\hline Serratia marcescens & +++ & +++ & +++ & ++ & ++++ & ++++ & ++++ & +++ \\
\hline
\end{tabular}

${ }^{*}$ Cefepime has activity against ampC-producing organisms; ${ }^{\dagger}$ Includes ciprofloxacin, levofloxacin, moxifloxacin (moxifloxacin has only moderate activity against Pseudomonas aeruginosa); ${ }^{\ddagger}$ Includes gentamicin, netilmicin, tobramycin, amikacin. - Poor activity; + Limited activity and/or resistance $\geq 15 \%$; ++ Moderate to good activity and/or resistance $10 \%$ to $14 \%$; +++ Very good activity and/or resistance 5\% to 9\%; ++++ Excellent activity and/or resistance $<5 \%$; ESBL Extended-spectrum beta-lactamase; NA Not available; Pip-tazo Pipercillin-tazobactam; Ticar-clav Ticarcillin-clavulanate. Data adapted from references 216,248-255

\section{APPENDIX 3}

In vitro antimicrobial activity against anaerobes associated with intra-abdominal infections

\begin{tabular}{|c|c|c|c|c|c|c|c|}
\hline Bacteria & Clindamycin & Cefoxitin & $\begin{array}{l}\text { Levofloxacin/ } \\
\text { moxifloxacin }\end{array}$ & $\begin{array}{c}\text { Imipenem/meropenem } \\
\text { Ertapenem }\end{array}$ & $\begin{array}{l}\text { Pip-tazol } \\
\text { ticar-clav }\end{array}$ & Metronidazole & Tigecycline \\
\hline Bacteroides fragilis & $+/++$ & ++ & $+/+++*$ & ++++ & ++++ & ++++ & ++++ \\
\hline Bacteroides fragilis group & + & ++ & + & ++++ & +++ & ++++ & ++++ \\
\hline Fusobacterium species & +++ & ++++ & $+/++$ & ++++ & ++++ & ++++ & ++++ \\
\hline Peptostreptococcus species & ++++ & ++++ & +++ & ++++ & ++++ & + & ++++ \\
\hline Clostridium perfringens & +++ & ++++ & +++ & ++++ & ++++ & +++ & +++ \\
\hline Clostridium difficile & $-1+$ & $-1+$ & ++ & + & ++ & +++ & ++++ \\
\hline Lactobacillus species & + & + & $+/++$ & $-1+$ & ++ & + & + \\
\hline Veillonella species & ++++ & + & + & +++ & ++ & +++ & + \\
\hline
\end{tabular}

*Moxifloxacin has good activity against Bacteroides fragilis. - Poor activity; + Limited activity and/or resistance $\geq 15 \%$; ++ Moderate to good activity and/or resistance $10 \%$ to $14 \%$; +++ Very good activity and/or resistance $5 \%$ to $9 \%$; ++++ Excellent activity and/or resistance <5\%; Pip-tazo Pipercillin-tazobactam; Ticar-clav Ticarcillin-clavulanate. Data adapted from references $85,86,256-258$ 


\section{APPENDIX 4}

Spectrum of activity of different antiseptic agents

\begin{tabular}{|c|c|c|c|c|c|}
\hline Agent & Mechanism of action & $\begin{array}{c}\text { Gram-positive } \\
\text { bacteria }\end{array}$ & $\begin{array}{c}\text { Gram-negative } \\
\text { Bacteria }\end{array}$ & Issues and toxicity & Indicated for surgical scrub \\
\hline \multirow[t]{2}{*}{ Alcohol } & Destroys proteins & $E$ & $E$ & Flammable & Yes \\
\hline & & & & Drying, no activity against spores & \\
\hline Iodophors/iodine & Oxidation by free iodine & $\mathrm{E}$ & G & $\begin{array}{l}\text { Allergy, rashes, inactivation by } \\
\text { blood and proteins }\end{array}$ & Yes \\
\hline $\begin{array}{l}\text { Chlorhexidine-based } \\
\text { agents }\end{array}$ & Cell membrane disruption & $E$ & G & Ototoxicity & Yes \\
\hline
\end{tabular}

E Excellent activity; G Good activity

\section{REFERENCES}

1. Solomkin JS, Mazuski JE, Baron EJ, et al. Guidelines for the selection of anti-infective agents for complicated intra-abdominal infections. Clin Infect Dis 2003;37:997-1005.

2. Krukowski ZH, Matheson NA. Ten-year computerized audit of infection after abdominal surgery. Br J Surg 1988;75:857-61.

3. Weiss G, Meyer F, Lippert H. Infectiological diagnostic problems in tertiary peritonitis. Langenbecks Arch Surg 2006;391:473-82.

4. Cheadle WG, Spain DA. The continuing challenge of intra-abdominal infection. Am J Surg 2003;186:15S-22S.

5. Christou NV, Barie PS, Dellinger EP, et al. Surgical Infection Society intra-abdominal infection study. Prospective evaluation of management techniques and outcome. Arch Surg 1993;128:193-8.

6. Nathens AB, Rotstein OD, Marshall JC. Tertiary peritonitis: Clinical features of a complex nosocomial infection. World J Surg 1998;22:158-63.

7. Malangoni MA, Inui T. Peritonitis - The Western experience. World J Emerg Surg 2006;1:25.

8. Marshall JC, Innes M. Intensive care unit management of intra-abdominal infection. Crit Care Med 2003;31:2228-37.

9. Wong PF, Gilliam AD, Kumar S, et al. Antibiotic regimens for secondary peritonitis of gastrointestinal origin in adults. Cochrane Database Syst Rev 2005:CD004539.

10. Kish MA. Guide to development of practice guidelines. Clin Infect Dis 2001;32:851-4.

11. de Meyrick J. The Delphi method and health resarch. Health Education 2003;103:7-16.

12. Bohnen J, Boulanger M, Meakins JL, et al. Prognosis in generalized peritonitis. Relation to cause and risk factors. Arch Surg 1983;118:285-90.

13. Mazuski JE, Sawyer RG, Nathens AB, et al. The Surgical Infection Society guidelines on antimicrobial therapy for intra-abdominal infections: Evidence for the recommendations. Surg Infect (Larchmt) 2002;3:175-233.

14. Garner JS, Jarvis WR, Emori TG, et al. CDC definitions for nosocomial infections, 1988. Am J Infect Control 1988;16:128-40.

15. Montravers P, Chalfine A, Gauzit R, et al. Clinical and therapeutic features of nonpostoperative nosocomial intra-abdominal infections. Ann Surg 2004;239:409-16.

16. Blot S, De Waele JJ. Critical issues in the clinical management of complicated intra-abdominal infections. Drugs 2005;65:1611-20.

17. Malangoni MA. Evaluation and management of tertiary peritonitis. Am Surg 2000;66:157-61.

18. Klevens RM, Edwards JR, Richards CL Jr, et al. Estimating health care-associated infections and deaths in U.S. hospitals, 2002. Public Health Rep 2007;122:160-6.

19. Buijk SL, Gyssens IC, Mouton JW, et al. Pharmacokinetics of ceftazidime in serum and peritoneal exudate during continuous versus intermittent administration to patients with severe intraabdominal infections. J Antimicrob Chemother 2002;49:121-8.

20. Christou NV. Predicting infectious morbidity in elective operations. Am J Surg 1993;165:52S-8S.

21. Marshall JC. Sepsis: Current status, future prospects. Curr Opin Crit Care 2004;10:250-64.

22. Trapnell JE, Duncan EH. Patterns of incidence in acute pancreatitis. Br Med J 1975;2:179-83.

23. McKay CJ, Evans S, Sinclair M, et al. High early mortality rate from acute pancreatitis in Scotland, 1984-1995. Br J Surg $1999 ; 86: 1302-5$.
24. Worning H. [Acute pancreatitis in Denmark]. Ugeskr Laeger 1994;156:2086-9.

25. Lankisch PG, Assmus C, Pflichthofer D, et al. Which etiology causes the most severe acute pancreatitis? Int J Pancreatol 1999;26:55-7.

26. Corfield AP, Cooper MJ, Williamson RC. Acute pancreatitis: A lethal disease of increasing incidence. Gut 1985;26:724-9.

27. NNIS. National Nosocomial Infections Surveillance (NNIS) System report, data summary from October 1986-April 1998. Am J Infect Control 1998;26:522-33.

28. Dellinger EP, Oreskovich MR, Wertz MJ, et al. Risk of infection following laparotomy for penetrating abdominal injury. Arch Surg 1984;119:20-7.

29. Goins WA, Rodriguez A, Joshi M, et al. Intra-abdominal abscess after blunt abdominal trauma. Ann Surg 1990;212:60-5.

30. Morales CH, Villegas MI, Villavicencio R, et al. Intra-abdominal infection in patients with abdominal trauma. Arch Surg 2004;139:1278-85.

31. Mulier S, Penninckx F, Verwaest C, et al. Factors affecting mortality in generalized postoperative peritonitis: Multivariate analysis in 96 patients. World J Surg 2003;27:379-84.

32. Dellinger EP, Wertz MJ, Meakins JL, et al. Surgical infection stratification system for intra-abdominal infection. Multicenter trial. Arch Surg 1985;120:21-9.

33. Wickel DJ, Cheadle WG, Mercer-Jones MA, et al. Poor outcome from peritonitis is caused by disease acuity and organ failure, not recurrent peritoneal infection. Ann Surg 1997;225:744-53.

34. Sturkenboom MC, Goettsch WG, Picelli G, et al. Inappropriate initial treatment of secondary intra-abdominal infections leads to increased risk of clinical failure and costs. Br J Clin Pharmacol 2005;60:438-43.

35. Krobot K, Yin D, Zhang Q, et al. Effect of inappropriate initial empiric antibiotic therapy on outcome of patients with community-acquired intra-abdominal infections requiring surgery. Eur J Clin Microbiol Infect Dis 2004;23:682-7.

36. Bare M, Castells X, Garcia A, et al. Importance of appropriateness of empiric antibiotic therapy on clinical outcomes in intra-abdominal infections. Int J Technol Assess Health Care 2006;22:242-8.

37. Mazuski JE. Clinical challenges and unmet needs in the management of complicated intra-abdominal infections. Surg Infect 2005;6:s49-s69.

38. Hynninen M, Wennervirta J, Leppaniemi A, et al. Organ dysfunction and long term outcome in secondary peritonitis. Langenbecks Arch Surg 2008;393:81-6.

39. Kulkarni SV, Naik AS, Subramanian N Jr. APACHE-II scoring system in perforative peritonitis. Am J Surg 2007;194:549-52.

40. Whittmann DH, Condon RE, Walker AP. Peritonitis and intraabdominal infection. In: Schwartz S, ed. Principles of Surgery, 6th edn. New York, McGraw-Hill, 1993:1449-83.

41. Bohnen JM, Mustard RA, Oxholm SE, et al. APACHE II score and abdominal sepsis. A prospective study. Arch Surg 1988;123:225-9.

42. Sorensen LT, Hemmingsen U, Kallehave F, et al. Risk factors for tissue and wound complications in gastrointestinal surgery. Ann Surg 2005;241:654-8.

43. Cruse PJ, Foord R. A five-year prospective study of 23,649 surgical wounds. Arch Surg 1973;107:206-10.

44. Burger JW, van 't RM, Jeekel J. Abdominal incisions: Techniques and postoperative complications. Scand J Surg 2002;91:315-21. 
45. Riou JP, Cohen JR, Johnson H Jr. Factors influencing wound dehiscence. Am J Surg 1992;163:324-30.

46. Wacha H, Hau T, Dittmer R, et al. Risk factors associated with intraabdominal infections: A prospective multicenter study. Peritonitis Study Group. Langenbecks Arch Surg 1999;384:24-32.

47. Ohmann C, Hau T. Prognostic indices in peritonitis. Hepatogastroenterology 1997;44:937-46.

48. Pacelli F, Doglietto GB, Alfieri S, et al. Prognosis in intra-abdominal infections. Multivariate analysis on 604 patients. Arch Surg 1996;131:641-5.

49. Solomkin JS, Dellinger EP, Christou NV, et al. Results of a multicenter trial comparing imipenem/cilastatin to tobramycin/ clindamycin for intra-abdominal infections. Ann Surg 1990;212:581-91.

50. Pitcher WD, Musher DM. Critical importance of early diagnosis and treatment of intra-abdominal infection. Arch Surg 1982;117:328-33

51. Hopkins JA, Lee JC, Wilson SE. Susceptibility of intra-abdominal isolates at operation: A predictor of postoperative infection. Am Surg 1993;59:791-6.

52. Christou NV, Turgeon P, Wassef R, et al. Management of intra-abdominal infections. The case for intraoperative cultures and comprehensive broad-spectrum antibiotic coverage. The Canadian Intra-abdominal Infection Study Group. Arch Surg 1996;131:1193-201.

53. McGilvray ID, Rotstein OD. Management of infection in the surgical patient: An update. Surg Technol Int 2003;11:39-43.

54. Carman RJ, Van Tassell RL, Wilkins TD. The normal intestinal microflora: Ecology, variability and stability. Vet Hum Toxicol 1993;35(Suppl 1):11-4.

55. Gorbach SL, Barza M, Giuliano M, et al. Colonization resistance of the human intestinal microflora: Testing the hypothesis in normal volunteers. Eur J Clin Microbiol Infect Dis 1988;7:98-102.

56. Hao WL, Lee YK. Microflora of the gastrointestinal tract: A review. Methods Mol Biol 2004;268:491-502.

57. DiNubile MJ, Chow JW, Satishchandran V, et al. Acquisition of resistant bowel flora during a double-blind randomized clinical trial of ertapenem versus piperacillin-tazobactam therapy for intraabdominal infections. Antimicrob Agents Chemother 2005;49:3217-21.

58. Hebuterne X. Gut changes attributed to ageing: Effects on intestinal microflora. Curr Opin Clin Nutr Metab Care 2003;6:49-54.

59. Nord CE, Kager L, Heimdahl A. Impact of antimicrobial agents on the gastrointestinal microflora and the risk of infections. Am J Med 1984;76:99-106.

60. Giamarellou H, Kanellakopoulou K. Bacteriologic and therapeutic considerations in intra-abdominal surgical infections. Anaerobe 1997;3:207-12.

61. Barie PS, Rotstein OD, Dellinger EP, et al. The cost-effectiveness of cefepime plus metronidazole versus imipenem/cilastatin in the treatment of complicated intra-abdominal infection. Surg Infect (Larchmt) 2004;5:269-80.

62. Kochhar P, Suvarna V, Duttagupta S, et al. Cost-effectiveness study comparing cefoperazone-sulbactam to a three-drug combination for treating intraabdominal infections in an Indian health-care setting. Value Health 2008;11(Suppl 1):S33-8.

63. Panhofer P, Izay B, Riedl M, et al. Age, microbiology and prognostic scores help to differentiate between secondary and tertiary peritonitis. Langenbecks Arch Surg 2009;394:265-71.

64. Delibegovic S. Pathophysiological changes in peritonitis. Med Arh 2007;61:109-13.

65. Monteiro MC, Danielou A, Piemont Y, et al. Bacterial cultures and empirical antimicrobial therapy for community-acquired secondary peritonitis. J Chir (Paris) 2007;144:486-91.

66. Shinagawa N, Tanaka K, Mikamo H, et al. Bacteria isolated from perforation peritonitis and their antimicrobial susceptibilities. Jpn J Antibiot 2007;60:206-20.

67. Pungpapong S, Alvarez S, Hellinger WC, et al. Peritonitis after liver transplantation: Incidence, risk factors, microbiology profiles, and outcome. Liver Transpl 2006;12:1244-52.

68. O'Hara AM, Shanahan F. The gut flora as a forgotten organ. EMBO Rep 2006; 7:688-93.

69. Guarner F. Enteric flora in health and disease. Digestion 2006;73(Suppl 1):5-12.

70. Chow JW, Satishchandran V, Snyder TA, et al. In vitro susceptibilities of aerobic and facultative gram-negative bacilli isolated from patients with intra-abdominal infections worldwide: The 2002 Study for Monitoring Antimicrobial Resistance Trends (SMART). Surg Infect (Larchmt) 2005;6:439-48.

71. Goldstein EJ, Snydman DR. Intra-abdominal infections: Review of the bacteriology, antimicrobial susceptibility and the role of ertapenem in their therapy. J Antimicrob Chemother 2004;53(Suppl 2):ii29-ii36.

72. Mai V, Morris JG Jr. Colonic bacterial flora: Changing understandings in the molecular age. J Nutr 2004;134:459-64.

73. Brook I. Microbiology and management of intra-abdominal infections in children. Pediatr Int 2003;45:123-9.

74. Brook I. Microbiology of polymicrobial abscesses and implications for therapy. J Antimicrob Chemother 2002;50:805-10.

75. Onderdonk AB, Weinstein WM, Sullivan NM, et al. Experimental intra-abdominal abscesses in rats: Quantitative bacteriology of infected animals. Infect Immun 1974;10:1256-9.

76. Weinstein WM, Onderdonk AB, Bartlett JG, et al. Experimental intra-abdominal abscesses in rats: Development of an experimental model. Infect Immun 1974;10:1250-5.

77. Condon RE. Microbiology of intraabdominal infection and contamination. Eur J Surg Suppl 1996;576:9-12.

78. Bohnen JM, Solomkin JS, Dellinger EP, et al. Guidelines for clinical care: Anti-infective agents for intra-abdominal infection. A Surgical Infection Society policy statement. Arch Surg 1992;127:83-9.

79. Laterre PF, Colardyn F, Delmee M, et al. Antimicrobial therapy for intra-abdominal infections: Guidelines from the Infectious Disease Advisory Board (IDAB). Acta Chir Belg 2006;106:2-21.

80. Goldstein EJ, Citron DM, Goldman PJ, et al. National hospital survey of anaerobic culture and susceptibility methods: III. Anaerobe 2008;14:68-72.

81. Dougherty SH. Antimicrobial culture and susceptibility testing has little value for routine management of secondary bacterial peritonitis. Clin Infect Dis 1997;25(Suppl 2):S258-S261.

82. Bilik R, Burnweit C, Shandling B. Is abdominal cavity culture of any value in appendicitis? Am J Surg 1998;175:267-70.

83. Kokoska ER, Silen ML, Tracy TF Jr, et al. The impact of intraoperative culture on treatment and outcome in children with perforated appendicitis. J Pediatr Surg 1999;34:749-53.

84. Falagas ME, Barefoot L, Griffith J, et al. Risk factors leading to clinical failure in the treatment of intra-abdominal or skin/soft tissue infectins. Eur J Clin Microbiol Infect Dis 1996;15:913-21.

85. Snydman DR, Jacobus NV, McDermott LA, et al. National survey on the susceptibility of Bacteroides fragilis group: Report and analysis of trends in the United States from 1997 to 2004. Antimicrob Agents Chemother 2007;51:1649-55.

86. Goldstein EJ, Citron DM, Vaidya SA, et al. In vitro activity of 11 antibiotics against 74 anaerobes isolated from pediatric intraabdominal infections. Anaerobe 2006;12:63-6.

87. Ulger TN, Celik C, Cakici O, et al. Antimicrobial susceptibilities of Bacteroides fragilis and Bacteroides thetaiotaomicron strains isolated from clinical specimens and human intestinal microbiota. Anaerobe 2004;10:255-9.

88. Paterson DL, Rossi F, Baquero F, et al. In vitro susceptibilities of aerobic and facultative Gram-negative bacilli isolated from patients with intra-abdominal infections worldwide: The 2003 Study for Monitoring Antimicrobial Resistance Trends (SMART). J Antimicrob Chemother 2005;55:965-73.

89. Rossi F, Baquero F, Hsueh PR, et al. In vitro susceptibilities of aerobic and facultatively anaerobic Gram-negative bacilli isolated from patients with intra-abdominal infections worldwide: 2004 results from SMART (Study for Monitoring Antimicrobial Resistance Trends). J Antimicrob Chemother 2006;58:205-10.

90. Seguin P, Laviolle B, Chanavaz C, et al. Factors associated with multidrug-resistant bacteria in secondary peritonitis: Impact on antibiotic therapy. Clin Microbiol Infect 2006;12:980-5.

91. Aldridge KE, Ashcraft D, O'Brien M, et al. Bacteremia due to Bacteroides fragilis group: Distribution of species, beta-lactamase production, and antimicrobial susceptibility patterns. Antimicrob Agents Chemother 2003;47:148-53.

92. Wybo I, Pierard D, Verschraegen I, et al. Third Belgian multicentre survey of antibiotic susceptibility of anaerobic bacteria. J Antimicrob Chemother 2007;59:132-9.

93. Neuhauser MM, Weinstein RA, Rydman R, et al. Antibiotic resistance among gram-negative bacilli in US intensive care units: Implications for fluoroquinolone use. JAMA 2003;289:885-8. 
94. MacDougall C, Powell JP, Johnson CK, et al. Hospital and community fluoroquinolone use and resistance in Staphylococcus aureus and Escherichia coli in 17 US hospitals. Clin Infect Dis 2005;41:435-40.

95. Poenaru D, De Santis M, Christou NV. Imipenem versus tobramycin - antianaerobe antibiotic therapy in intra-abdominal infections. Can J Surg 1990;33:415-22.

96. Brismar B, Malmborg AS, Tunevall G, et al. Piperacillin-tazobactam versus imipenem-cilastatin for treatment of intra-abdominal infections. Antimicrob Agents Chemother 1992;36:2766-73.

97. Geroulanos S, Marathias K, Kriaras J, et al. Cephalosporins in surgical prophylaxis. J Chemother 2001;13 Spec No 1:23-6.

98. Babinchak T, Ellis-Grosse E, Dartois N, et al. The efficacy and safety of tigecycline for the treatment of complicated intra-abdominal infections: Analysis of pooled clinical trial data. Clin Infect Dis 2005;41(Suppl 5):S354-S367.

99. Condon RE, Walker AP, Sirinek KR, et al. Meropenem versus tobramycin plus clindamycin for treatment of intraabdominal infections: Results of a prospective, randomized, double-blind clinical trial. Clin Infect Dis 1995;21:544-50.

100. Dougherty SH, Sirinek KR, Schauer PR, et al. Ticarcillin/ clavulanate compared with clindamycin/gentamicin (with or without ampicillin) for the treatment of intra-abdominal infections in pediatric and adult patients. Am Surg 1995;61:297-303.

101. Solomkin JS, Wilson SE, Christou NV, et al. Results of a clinical trial of clinafloxacin versus imipenem/cilastatin for intraabdominal infections. Ann Surg 2001;233:79-87.

102. Malangoni MA, Song J, Herrington J, et al. Randomized controlled trial of moxifloxacin compared with piperacillin-tazobactam and amoxicillin-clavulanate for the treatment of complicated intraabdominal infections. Ann Surg 2006;244:204-11.

103. Solomkin JS, Reinhart HH, Dellinger EP, et al. Results of a randomized trial comparing sequential intravenous/oral treatment with ciprofloxacin plus metronidazole to imipenem/cilastatin for intra-abdominal infections. The Intra-Abdominal Infection Study Group. Ann Surg 1996;223:303-15.

104. Barie PS, Vogel SB, Dellinger EP, et al. A randomized, double-blind clinical trial comparing cefepime plus metronidazole with imipenem-cilastatin in the treatment of complicated intraabdominal infections. Cefepime Intra-abdominal Infection Study Group. Arch Surg 1997;132:1294-302.

105. Ohlin B, Cederberg A, Forssell H, et al. Piperacillin/tazobactam compared with cefuroxime/metronidazole in the treatment of intraabdominal infections. Eur J Surg 1999;165:875-84.

106. Heuer JG, Zhang T, Zhao J, et al. Adoptive transfer of in vitrostimulated $\mathrm{CD} 4+\mathrm{CD} 25+$ regulatory $\mathrm{T}$ cells increases bacterial clearance and improves survival in polymicrobial sepsis. J Immunol 2005;174:7141-6

107. Chung DR, Kasper DL, Panzo RJ, et al. CD4+ T cells mediate abscess formation in intra-abdominal sepsis by an IL-17-dependent mechanism. J Immunol 2003;170:1958-63.

108. Finlay-Jones JJ, Davies KV, Sturm LP, et al. Inflammatory processes in a murine model of intra-abdominal abscess formation. J Leukoc Biol 1999;66:583-7.

109. Chong AJ, Dellinger EP. Current treatment of intraabdominal infections. Surg Technol Int 2005;14:29-33.

110. Cohn SM, Lipsett PA, Buchman TG, et al. Comparison of intravenous/oral ciprofloxacin plus metronidazole versus piperacillin/tazobactam in the treatment of complicated intraabdominal infections. Ann Surg 2000;232:254-62.

111. Sitges-Serra A, Lopez MJ, Girvent M, et al. Postoperative enterococcal infection after treatment of complicated intra-abdominal sepsis. Br J Surg 2002;89:361-7.

112. Burnett RJ, Haverstock DC, Dellinger EP, et al. Definition of the role of enterococcus in intraabdominal infection: Analysis of a prospective randomized trial. Surgery 1995;118:716-21.

113. Malangoni MA. Contributions to the management of intraabdominal infections. Am J Surg 2005;190:255-9.

114. Bohnen JM. Operative management of intra-abdominal infections. Infect Dis Clin North Am 1992;6:511-23.

115. Oliva ME, Rekha A, Yellin A, et al. A multicenter trial of the efficacy and safety of tigecycline versus imipenem/cilastatin in patients with complicated intra-abdominal infections. BMC Infect Dis 2005;5:88-99.

116. Basoli A, Chirletti P, Cirino E, et al. A prospective, double-blind, multicenter, randomized trial comparing ertapenem 3 vs $>$ or $=5$ days in community-acquired intraabdominal infection. J Gastrointest Surg 2008;12:592-600.

117. Kempf P, Bauernfeind A, Muller A, et al. Meropenem monotherapy versus cefotaxime plus metronidazole combination treatment for serious intra-abdominal infections. Infection 1996;24:473-9.

118. Jauregui LE, Appelbaum PC, Fabian TC, et al. A randomized clinical study of cefoperazone and sulbactam versus gentamicin and clindamycin in the treatment of intra-abdominal infections. J Antimicrob Chemother 1990;25:423-33.

119. Berne TV, Yellin AW, Appleman MD, et al. Antibiotic management of surgically treated gangrenous or perforated appendicitis. Comparison of gentamicin and clindamycin versus cefamandole versus cefoperazone. Am J Surg 1982;144:8-13.

120. Garbino J, Villiger P, Caviezel A, et al. A randomized prospective study of cefepime plus metronidazole with imipenem-cilastatin in the treatment of intra-abdominal infections. Infection 2007;35:161-6.

121. Merlino JI, Malangoni MA, Smith CM, et al. Prospective randomized trials affect the outcomes of intraabdominal infection. Ann Surg 2001;233:859-66.

122. Meehan TP, Weingarten SR, Holmboe ES, et al. A statewide initiative to improve the care of hospitalized pneumonia patients: The Connecticut Pneumonia Pathway Project. Am J Med 2001;111:203-10.

123. Welker JA, Huston M, McCue JD. Antibiotic timing and errors in diagnosing pneumonia. Arch Intern Med 2008;168:351-6.

124. Chow AW. Gastrointestinal and intraabdominal infections. In: Ronald AR, Low DE, eds. Fluoroquinolone Antibiotics, 1st edn. Basel, Birkhauser Verlag: 2003;137-66.

125. Madan AK. Use of ciprofloxacin in the treatment of hospitalized patients with intra-abdominal infections. Clin Ther 2004;26:1564-77.

126. Matthaiou DK, Peppas G, Bliziotis IA, et al. Ciprofloxacin/ metronidazole versus beta-lactam-based treatment of intraabdominal infections: A meta-analysis of comparative trials. Int J Antimicrob Agents 2006;28:159-65.

127. Wacha $\mathrm{H}$, Warren $\mathrm{B}$, Bassaris $\mathrm{H}$, et al. Comparison of sequential intravenous/oral ciprofloxacin plus metronidazole with intravenous ceftriaxone plus metronidazole for treatment of complicated intra-abdominal infections. Surg Infect (Larchmt) 2006;7:341-54.

128. Walters DJ, Solomkin JS, Paladino JA. Cost effectiveness of ciprofloxacin plus metronidazole versus imipenem-cilastatin in the treatment of intra-abdominal infections. Pharmacoeconomics 1999;16:551-61.

129. Donahue PE, Smith DL, Yellin AE, et al. Trovafloxacin in the treatment of intra-abdominal infections: Results of a double-blind, multicenter comparison with imipenem/cilastatin. Am J Surg 1998;176(Suppl 6A):53S-61S

130. Lockhart SR, Abramson MA, Beekmann SE, et al. Antimicrobial resistance among Gram-negative bacilli causing infections in intensive care unit patients in the United States between 1993 and 2004. J Clin Microbiol 2007;45:3352-9.

131. Kern WV, Klose K, Jellen-Ritter AS, et al. Fluoroquinolone resistance of Escherichia coli at a cancer center: Epidemiologic evolution and effects of discontinuing prophylactic fluoroquinolone use in neutropenic patients with leukemia. Eur J Clin Microbiol Infect Dis 2005;24:111-8.

132. Kaye KS, Kanafani ZA, Dodds AE, et al. Differential effects of levofloxacin and ciprofloxacin on the risk for isolation of quinolone-resistant Pseudomonas aeruginosa. Antimicrob Agents Chemother 2006;50:2192-6.

133. Bailey JA, Virgo KS, DiPiro JT, et al. Aminoglycosides for intraabdominal infection: Equal to the challenge? Surg Infect (Larchmt) 2002;3:315-35.

134. Wirtz M, Kleeff J, Swoboda S, et al. Moxifloxacin penetration into human gastrointestinal tissues. J Antimicrob Chemother 2004;53:875-7.

135. Stass H, Rink AD, Delesen H, et al. Pharmacokinetics and peritoneal penetration of moxifloxacin in peritonitis. J Antimicrob Chemother 2006;58:693-6.

136. Hoban DJ, Bouchillon SK, Johnson BM, et al. In vitro activity of tigecycline against 6792 Gram-negative and Gram-positive clinical isolates from the global Tigecycline Evaluation and Surveillance Trial (TEST Program, 2004). Diagn Microbiol Infect Dis 2005;52:215-27.

137. Guyot A, Layer G. MRSA - 'bug-bear' of a surgical practice: Reducing the incidence of MRSA surgical site infections. Ann R Coll Surg Engl 2006;88:222-3. 
138. Rezende NA, Blumberg HM, Metzger BS, et al. Risk factors for methicillin-resistance among patients with Staphylococcus aureus bacteremia at the time of hospital admission. Am J Med Sci 2002;323:117-23

139. Fierobe L, Decre D, Muller C, et al. Methicillin-resistant Staphylococcus aureus as a causative agent of postoperative intraabdominal infection: Relation to nasal colonization. Clin Infect Dis 1999;29:1231-8.

140. Patel M, Kumar RA, Stamm AM, et al. USA300 genotype community-associated methicillin-resistant Staphylococcus aureus as a cause of surgical site infections. J Clin Microbiol 2007;45:3431-3.

141. Gemmell CG, Edwards DI, Fraise AP, et al. Guidelines for the prophylaxis and treatment of methicillin-resistant Staphylococcus aureus (MRSA) infections in the UK. J Antimicrob Chemother 2006;57:589-608.

142. Rohrborn A, Wacha H, Schoffel U, et al. Coverage of enterococci in community acquired secondary peritonitis: Results of a randomized trial. Surg Infect (Larchmt) 2000;1:95-107

143. Harbarth S, Uckay I. Are there patients with peritonitis who require empiric therapy for enterococcus? Eur J Clin Microbiol Infect Dis 2004:23:73-7.

144. Mean M, Marchetti O, Calandra T. Bench-to-bedside review: Candida infections in the intensive care unit. Crit Care 2008;12:204-12.

145. Montravers P, Dupont H, Gauzit R, et al. Candida as a risk factor for mortality in peritonitis. Crit Care Med 2006;34:646-52.

146. Dupont H, Paugam-Burtz C, Muller-Serieys C, et al. Predictive factors of mortality due to polymicrobial peritonitis with Candida isolation in peritoneal fluid in critically ill patients. Arch Surg 2002;137:1341-6.

147. Piarroux R, Grenouillet F, Balvay P, et al. Assessment of preemptive treatment to prevent severe candidiasis in critically ill surgical patients. Crit Care Med 2004;32:2443-9.

148. Tsuruta R, Mizuno H, Kaneko T, et al. Preemptive therapy in nonneutropenic patients with Candida infection using the Japanese guidelines. Ann Pharmacother 2007;41:1137-43.

149. Eggimann P, Francioli P, Bille J, et al. Fluconazole prophylaxis prevents intra-abdominal candidiasis in high-risk surgical patients. Crit Care Med 1999;27:1066-72

150. Schein M, Assalia A, Bachus H. Minimal antibiotic therapy after emergency abdominal surgery: A prospective study. Br J Surg 1994:81:989-91.

151. Smith JA, Bell GA, Murphy J, et al. Evaluation of the use of a protocol in the antimicrobial treatment of intra-abdominal sepsis. J Hosp Infect 1985;6:60-4.

152. Taylor E, Dev V, Shah D, et al. Complicated appendicitis: Is there a minimum intravenous antibiotic requirement? A prospective randomized trial. Am Surg 2000;66:887-90.

153. Marshall JC. Intra-abdominal infections. Microbes Infect 2004;6:1015-25.

154. Colodner R, Rock W, Chazan B, et al. Risk factors for the development of extended-spectrum beta-lactamase-producing bacteria in nonhospitalized patients. Eur J Clin Microbiol Infect Dis 2004;23:163-7.

155. Kang CI, Kim SH, Park WB, et al. Bloodstream infections due to extended-spectrum beta-lactamase-producing Escherichia coli and Klebsiella pneumoniae: Risk factors for mortality and treatment outcome, with special emphasis on antimicrobial therapy. Antimicrob Agents Chemother 2004;48:4574-81.

156. Schneider CP, Seyboth C, Vilsmaier M, et al. Prognostic factors in critically ill patients suffering from secondary peritonitis: A retrospective, observational, survival time analysis. World J Surg 2009;33:34-43.

157. Suding PN, Orrico RP, Johnson SB, et al. Concordance of interrater assessments of surgical methods to achieve source control of intraabdominal infections. Am J Surg 2008;196:70-3.

158. Kumar RR, Kim JT, Haukoos JS, et al. Factors affecting the successful management of intra-abdominal abscesses with antibiotics and the need for percutaneous drainage. Dis Colon Rectum 2006;49:183-9.

159. Siewert B, Tye G, Kruskal J, et al. Impact of CT-guided drainage in the treatment of diverticular abscesses: Size matters. Am J Roentgenol 2006;186:680-6.

160. Cinat ME, Wilson SE, Din AM. Determinants for successful percutaneous image-guided drainage of intra-abdominal abscess. Arch Surg 2002;137:845-9.
161. Cheng D, Nagata KT, Yoon HC. Randomized prospective comparison of alteplase versus saline solution for the percutaneous treatment of loculated abdominopelvic abscesses. J Vasc Interv Radiol 2008;19:906-11.

162. Beland MD, Gervais DA, Levis DA, et al. Complex abdominal and pelvic abscesses: Efficacy of adjunctive tissue-type plasminogen activator for drainage. Radiology 2008;247:567-73.

163. Bretagnol F, Pautrat K, Mor C, et al. Emergency laparoscopic management of perforated sigmoid diverticulitis: A promising alternative to more radical procedures. J Am Coll Surg 2008;206:654-7.

164. Franklin ME Jr, Portillo G, Trevino JM, et al. Long-term experience with the laparoscopic approach to perforated diverticulitis plus generalized peritonitis. World J Surg 2008;32:1507-11.

165. Taylor CJ, Layani L, Ghusn MA, et al. Perforated diverticulitis managed by laparoscopic lavage. ANZ J Surg 2006;76:962-5.

166. Myers E, Hurley M, O'Sullivan GC, et al. Laparoscopic peritoneal lavage for generalized peritonitis due to perforated diverticulitis. Br J Surg 2008;95:97-101.

167. Baril NB, Ralls PW, Wren SM, et al. Does an infected peripancreatic fluid collection or abscess mandate operation? Ann Surg 2000;231:361-7.

168. Olah A, Belagyi T, Bartek P, et al. Alternative treatment modalities of infected pancreatic necrosis. Hepatogastroenterology 2006;53:603-7.

169. Carter R. Percutaneous management of necrotizing pancreatitis. HPB (Oxford) 2007;9:235-9.

170. Loveday BP, Mittal A, Phillips A, et al. Minimally invasive management of pancreatic abscess, pseudocyst, and necrosis: A systematic review of current guidelines. World J Surg 2008;32:2383-94.

171. Lee JK, Kwak KK, Park JK, et al. The efficacy of nonsurgical treatment of infected pancreatic necrosis. Pancreas 2007;34:399-404.

172. Walker J, Criddle LM. Pathophysiology and management of abdominal compartment syndrome. Am J Crit Care 2003;12:367-71.

173. Malbrain ML, De laet I, Viaene D, et al. In vitro validation of a novel method for continuous intra-abdominal pressure monitoring. Intensive Care Med 2008;34:740-5.

174. Pelosi P, Quintel M, Malbrain ML. Effect of intra-abdominal pressure on respiratory mechanics. Acta Clin Belg Suppl 2007;1:78-88.

175. De Laet I, Citerio G, Malbrain ML. The influence of intraabdominal hypertension on the central nervous system: Current insights and clinical recommendations, is it all in the head? Acta Clin Belg Suppl 2007;1:89-97.

176. Cheatham ML, Malbrain ML. Cardiovascular implications of abdominal compartment syndrome. Acta Clin Belg Suppl 2007;1:98-112.

177. Diebel LN, Dulchavsky SA, Brown WJ. Splanchnic ischemia and bacterial translocation in the abdominal compartment syndrome. J Trauma 1997;43:852-5.

178. Diebel LN, Liberati DM, Dulchavsky SA, et al. An in vitro model to assess mucosal immune function and bacterial translocation. J Surg Res 1997;69:178-82.

179. Malbrain ML, Cheatham ML, Kirkpatrick A, et al. Results from the International Conference of Experts on Intra-abdominal Hypertension and Abdominal Compartment Syndrome. I. Definitions. Intensive Care Med 2006;32:1722-32

180. Cheatham ML, White MW, Sagraves SG, et al. Abdominal perfusion pressure: A superior parameter in the assessment of intra-abdominal hypertension. J Trauma 2000;49:621-6.

181. Malbrain ML, Deeren D, De Potter TJ. Intra-abdominal hypertension in the critically ill: It is time to pay attention. Curr Opin Crit Care 2005;11:156-71.

182. Malbrain ML, Chiumello D, Pelosi P, et al. Prevalence of intra-abdominal hypertension in critically ill patients: A multicentre epidemiological study. Intensive Care Med 2004;30:822-9.

183. Malbrain ML, Chiumello D, Pelosi P, et al. Incidence and prognosis of intraabdominal hypertension in a mixed population of critically ill patients: A multiple-center epidemiological study. Crit Care Med 2005;33:315-22.

184. Bodnar Z, Szentkereszty Z, Sipka S, et al. The epidemiology of intra-abdominal hypertension and abdominal compartment 
syndrome in critically ill general surgical patients. Acta Clin Belg Suppl 2007;62:S250.

185. De Waele JJ, Hoste E, Blot SI, et al. Intra-abdominal hypertension in patients with severe acute pancreatitis. Crit Care 2005;9:R452-R457.

186. Rosas JM, Soto SN, Aracil JS, et al. Intra-abdominal pressure as a marker of severity in acute pancreatitis. Surgery 2007;141:173-8.

187. Pupelis G, Austrums E, Snippe K, et al. Clinical significance of increased intraabdominal pressure in severe acute pancreatitis. Acta Chir Belg 2002;102:71-4.

188. Leppaniemi A, Kemppainen E. Recent advances in the surgical management of necrotizing pancreatitis. Curr Opin Crit Care 2005;11:349-52.

189. Plantefeve G, Hellmann R, Pajot O, et al. Abdominal compartment syndrome and intraabdominal sepsis: Two of the same kind? Acta Clin Belg Suppl 2007;1:162-7.

190. Busani S, Soccorsi MC, Poma C, et al. Intra-abdominal hypertension in nonelective surgery: A preliminary report. Transplant Proc 2006;38:836-7.

191. De Waele JJ, Hesse UJ. Life saving abdominal decompression in a patient with severe acute pancreatitis. Acta Chir Belg 2005;105:96-8.

192. Gecelter G, Fahoum B, Gardezi S, et al. Abdominal compartment syndrome in severe acute pancreatitis: An indication for a decompressing laparotomy? Dig Surg 2002;19:402-4.

193. Wong K, Summerhays CF. Abdominal compartment syndrome: A new indication for operative intervention in severe acute pancreatitis. Int J Clin Pract 2005;59:1479-81.

194. Oda S, Hirasawa H, Shiga H, et al. Management of intra-abdominal hypertension in patients with severe acute pancreatitis with continuous hemodiafiltration using a polymethyl methacrylate membrane hemofilter. Ther Apher Dial 2005;9:355-61.

195. Leppaniemi AK, Hienonen PA, Siren JE, et al. Treatment of abdominal compartment syndrome with subcutaneous anterior abdominal fasciotomy in severe acute pancreatitis. World J Surg 2006;30:1922-4.

196. Malbrain ML, De laet I, Cheatham M. Consensus conference definitions and recommendations on intra-abdominal hypertension (IAH) and the abdominal compartment syndrome (ACS) - the long road to the final publications, how did we get there? Acta Clin Belg Suppl 2007;1:44-59.

197. Cheatham ML, Malbrain ML, Kirkpatrick A, et al. Results from the International Conference of Experts on Intra-abdominal Hypertension and Abdominal Compartment Syndrome. II. Recommendations. Intensive Care Med 2007;33:951-62.

198. Balogh Z, De Waele JJ, Malbrain ML. Continuous intra-abdominal pressure monitoring. Acta Clin Belg Suppl 2007;1:26-32.

199. Hedrick TL, Anastacio MM, Sawyer RG. Prevention of surgical site infections. Expert Rev Anti Infect Ther 2006;4:223-33.

200. Mangram AJ, Horan TC, Pearson ML, et al. Guideline for prevention of surgical site infection, 1999. Hospital Infection Control Practices Advisory Committee. Infect Control Hosp Epidemiol 1999;20:250-78.

201. Haley RW, Culver DH, White JW, et al. The efficacy of infection surveillance and control programs in preventing nosocomial infections in US hospitals. Am J Epidemiol 1985;121:182-205.

202. Culver DH, Horan TC, Gaynes RP, et al. Surgical wound infection rates by wound class, operative procedure, and patient risk index. National Nosocomial Infections Surveillance System. Am J Med 1991;91:152S-7S.

203. Haley RW, Culver DH, Morgan WM, et al. Identifying patients at high risk of surgical wound infection. A simple multivariate index of patient susceptibility and wound contamination. Am J Epidemiol 1985;121:206-15.

204. Zerr KJ, Furnary AP, Grunkemeier GL, et al. Glucose control lowers the risk of wound infection in diabetics after open heart operations. Ann Thorac Surg 1997;63:356-61.

205. Jones JK, Triplett RG. The relationship of cigarette smoking to impaired intraoral wound healing: A review of evidence and implications for patient care. J Oral Maxillofac Surg 1992;50:237-9.

206. American Institute of Architects. Guidelines for design and construction of hospital and health care facilities. 1996. Washington, DC, American Institute of Architects Press.

207. Nichols RL. The operating room. In: Bennett JV, Brachman PS, eds. Hospital Infections. Boston: Little Brown \& Co, 1992;461-73.
208. Larson E. Guideline for use of topical antimicrobial agents. Am J Infect Control 1988;16:253-66.

209. The Medical Letter. Antimicrobial prophylaxis for surgery: treatment guidelines. Med Lett 2004;2:27-32.

210. Classen DC, Evans RS, Pestotnik SL, et al. The timing of prophylactic administration of antibiotics and the risk of surgical-wound infection. N Engl J Med 1992;326:281-6.

211. Simor AE, Ofner-Agostini M, Bryce E, et al. The evolution of methicillin-resistant Staphylococcus aureus in Canadian hospitals: 5 years of national surveillance. CMAJ 2001;165:21-6.

212. Ruef C. Epidemiology and clinical impact of glycopeptide resistance in Staphylococcus aureus. Infection 2004:32:315-27.

213. Muto CA, Jernigan JA, Ostrowsky BE, et al. SHEA guideline for preventing nosocomial transmission of multidrug-resistant strains of Staphylococcus aureus and enterococcus. Infect Control Hosp Epidemiol 2003;24:362-86.

214. Pittet D. Improving adherence to hand hygiene practice: A multidisciplinary approach. Emerg Infect Dis 2001;7:234-40.

215. Zhanel GG, Lam A, Schweizer F, et al. Ceftobiprole: A review of a broad-spectrum and anti-MRSA cephalosporin. Am J Clin Dermatol 2008;9:245-54.

216. Zhanel GG, Wiebe R, Dilay L, et al. Comparative review of the carbapenems. Drugs 2007;67:1027-52.

217. Walker AP, Nichols RL, Wilson RF, et al. Efficacy of a betalactamase inhibitor combination for serious intraabdominal infections. Ann Surg 1993;217:115-21.

218. Study Group of Intra-abdominal Infections. A randomized controled trial of ampicillin plus sulbactam versus gentamicin and clindamycin in the treatment of intraabdominal infections: A preliminary report. Rev Infect Dis 1986;8(Suppl 5):s583-s588.

219. Yellin AE, Heseltine PN, Berne TV, et al. The role of Pseudomonas species in patients treated with ampicillin and Sulbactam for gangrenous and perforated appendicitis. Surg Gynecol Obstet 1985;161:303-7.

220. Allo MD, Bennion RS, Kathir K, et al. Ticarcillin/clavulanate versus imipenem/cilistatin for the treatment of infections associated with gangrenous and perforated appendicitis. Am Surg 1999;65:99-104.

221. Najem AZ, Kaminski ZC, Spillert CR, et al. Comparative study of parenteral piperacillin and cefoxitin in the treatment of surgical infections of the abdomen. Surg Gynecol Obstet 1983;157:423-5.

222. Namias N, Solomkin JS, Jensen EH, et al. Randomized, multicenter, double-blind study of efficacy, safety, and tolerability of intravenous ertapenem versus piperacillin/tazobactam in treatment of complicated intra-abdominal infections in hospitalized adults. Surg Infect (Larchmt) 2007;8:15-28.

223. Paakkonen M, Alhava EM, Huttunen R, et al. Piperacillin compared with cefuroxime plus metronidazole in diffuse peritonitis. Eur J Surg 1991;157:535-7.

224. Shyr YM, Lui WY, Su CH, et al. Piperacillin/tazobactam in comparison with clindamycin plus gentamicin in the treatment of intra-abdominal infections. Zhonghua Yi Xue Za Zhi (Taipei) 1995; $56: 102-8$.

225. Dupont H, Carbon C, Carlet J. Monotherapy with a broad-spectrum beta-lactam is as effective as its combination with an aminoglycoside in treatment of severe generalized peritonitis: A multicenter randomized controlled trial. The Severe Generalized Peritonitis Study Group. Antimicrob Agents Chemother 2000;44:2028-33.

226. Investigators of the Piperacillin/Tazobactam Intra-abdominal Infection Study Group. Results of the North American trial of piperacillin/tazobactam compared with clindamycin and gentamicin in the treatment of severe intra-abdominal infections. Eur J Surg Suppl 1994;573:61-6.

227. Jaccard C, Troillet N, Harbarth S, et al. Prospective randomized comparison of imipenem-cilastatin and piperacillin-tazobactam in nosocomial pneumonia or peritonitis. Antimicrob Agents Chemother 1998;42:2966-72.

228. Solomkin JS, Yellin AE, Rotstein OD, et al. Ertapenem versus piperacillin/tazobactam in the treatment of complicated intraabdominal infections: Results of a double-blind, randomized comparative phase III trial. Ann Surg 2003;237:235-45.

229. Eckhauser FE, Knol JA, Raper SE, et al. Efficacy of two comparative antibiotic regimens in the treatment of serious intra-abdominal infections: Results of a multicenter study. Clin Ther 1992;14:97-109. 
230. Gonzenbach HR, Simmen HP, Amgwerd R. Imipenem (N-Fthienamycin) versus netilmicin plus clindamycin. A controlled and randomized comparison in intra-abdominal infections. Ann Surg 1987;205:271-5.

231. Angeras MH, Darle N, Hamnstrom K, et al. A comparison of imipenem/cilastatin with the combination of cefuroxime and metronidazole in the treatment of intra-abdominal infections. Scand J Infect Dis 1996;28:513-8.

232. de Groot HG, Hustinx PA, Lampe AS, et al. Comparison of imipenem/cilastatin with the combination of aztreonam and clindamycin in the treatment of intra-abdominal infections. J Antimicrob Chemother 1993;32:491-500.

233. Brismar B, Malmborg AS, Tunevall G, et al. Meropenem versus imipenem/cilastatin in the treatment of intra-abdominal infections. J Antimicrob Chemother 1995;35:139-48.

234. Basoli A, Meli EZ, Mazzocchi P, et al. Imipenem/cilastatin (1.5 g daily) versus meropenem ( $3.0 \mathrm{~g}$ daily) in patients with intraabdominal infections: Results of a prospective, randomized, multicentre trial. Scand J Infect Dis 1997;29:503-8.

235. Kanellakopoulou K, Giamarellou H, Papadothomakos P, et al. Meropenem versus imipenem/cilastatin in the treatment of intraabdominal infections requiring surgery. Eur J Clin Microbiol Infect Dis 1993;12:449-53.

236. Zanetti G, Harbarth SJ, Trampuz A, et al. Meropenem ( $1.5 \mathrm{~g} /$ day $)$ is as effective as imipenem/cilastatin ( $2 \mathrm{~g} /$ day $)$ for the treatment of moderately severe intra-abdominal infections. Int J Antimicrob Agents 1999;11:107-13.

237. Berne TV, Yellin AE, Appleman MD, et al. Meropenem versus tobramycin with clindamycin in the antibiotic management of patients with advanced appendicitis. J Am Coll Surg 1996;182:403-7.

238. Yellin AE, Hassett JM, Fernandez A, et al. Ertapenem monotherapy versus combination therapy with ceftriaxone plus metronidazole for treatment of complicated intra-abdominal infections in adults. Int J Antimicrob Agents 2002;20:165-73.

239. Hopkins JA, Wilson SE, Bobey DG. Adjunctive antimicrobial therapy for complicated appendicitis: Bacterial overkill by combination therapy. World J Surg 1994;18:933-8.

240. Malangoni MA, Condon RE, Spiegel CA. Treatment of intra-abdominal infections is appropriate with single-agent or combination antibiotic therapy. Surgery 1985;98:648-55.

241. Busuttil RW, McGrattan MA, Freischlag J. A comparative study of cefamandole versus gentamicin plus clindamycin in the treatment of documented or suspected bacterial peritonitis. Surg Gynecol Obstet 1984;158:1-8.

242. Tornqvist A, Forsgren A, Leandoer L, et al. Antibiotic treatment during surgery for diffuse peritonitis: A prospective randomized study comparing the effects of cefuroxime and of a cefuroxime and metronidazole combination. Br J Surg 1985;72:261-4.

243. Greenberg RN, Cayavec P, Danko LS, et al. Comparison of cefoperazone plus sulbactam with clindamycin plus gentamicin as treatment for intra-abdominal infections. J Antimicrob Chemother 1994;34:391-401.
244. Berne TV, Yellin AE, Appleman MD, et al. Surgically treated gangrenous or perforated appendicitis. A comparison of aztreonam and clindamycin versus gentamicin and clindamycin. Ann Surg 1987;205:133-7.

245. Berne TV, Yellin AE, Appleman MD, et al. A clinical comparison of cefepime and metronidazole versus gentamicin and clindamycin in the antibiotic management of surgically treated advanced appendicitis. Surg Gynecol Obstet 1993;177(Suppl):18-22.

246. Bubrick MP, Heim-Duthoy KL, Yellin AE, et al. Ceftazidime/ clindamycin versus tobramycin/clindamycin in the treatment of intra-abdominal infections. Am Surg 1990;56:613-7.

247. Starakis I, Karravias D, Asimakopoulos C, et al. Results of a prospective, randomized, double blind comparison of the efficacy and the safety of sequential ciprofloxacin (intravenous/ oral) + metronidazole (intravenous/oral) with ceftriaxone (intravenous)+metronidazole (intravenous/oral) for the treatment of intra-abdominal infections. Int J Antimicrob Agents 2003;21:49-57.

248. Zhanel GG, Karlowsky JA, Rubinstein E, et al. Tigecycline: A novel glycylcycline antibiotic. Expert Rev Anti Infect Ther 2006;4:9-25.

249. Zhanel GG, Johanson C, Embil JM, et al. Ertapenem: Review of a new carbapenem. Expert Rev Anti Infect Ther 2005;3:23-39.

250. Zhanel GG, Laing NM, Nichol KA, et al. Antibiotic activity against urinary tract infection (UTI) isolates of vancomycinresistant enterococci (VRE): Results from the 2002 North American Vancomycin Resistant Enterococci Susceptibility Study (NAVRESS). J Antimicrob Chemother 2003;52:382-8.

251. Zhanel GG, Ennis K, Vercaigne L, et al. A critical review of the fluoroquinolones: Focus on respiratory infections. Drugs 2002;62:13-59.

252. Zhanel GG, Shroeder C, Vercaigne L, et al. A critical review of oxazolidinones: An alternative or replacement for glycopeptides and streptogramins? Can J Infect Dis 2001;12:379-90.

253. Zhanel GG, Simor A, Vercaigne L, et al. Imipenem/meropenem: Review of in-vitro activity, pharmacokinetics, clinical trials and adverse effects. Can J Infect Dis 1998;44:391-6.

254. Gin A, Dilay L, Karlowsky JA, et al. Piperacillin-tazobactam: A beta-lactam/beta-lactamase inhibitor combination. Expert Rev Anti Infect Ther 2007;5:365-83.

255. Clinical and Laboratory Standards Institute (CLSI). Performance standards for antimicrobial susceptibility tesing. 15th Informational Supplement M100-S15. 2005. Wayne, PA, Clinical and Laboratory Standards Institute.

256. Goldstein EJ, Citron DM, Warren YA, et al. In vitro activity of moxifloxacin against 923 anaerobes isolated from human intra-abdominal infections. Antimicrob Agents Chemother 2006;50:148-55.

257. Aldridge KE, O'Brien M. In vitro susceptibilities of the Bacteroides fragilis group species: Change in isolation rates significantly affects overall susceptibility data. J Clin Microbiol 2002;40:4349-52.

258. Roberts SA, Shore KP, Paviour SD, et al. Antimicrobial susceptibility of anaerobic bacteria in New Zealand: 1999-2003. J Antimicrob Chemother 2006;57:992-8. 


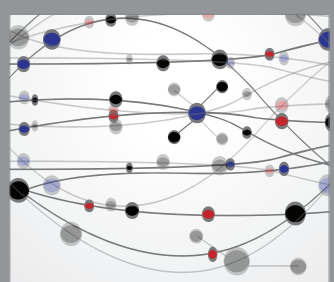

The Scientific World Journal
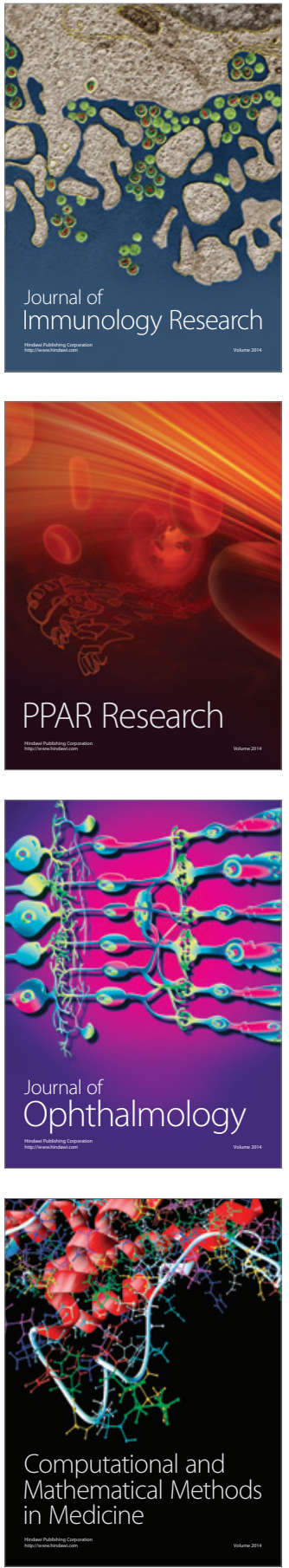

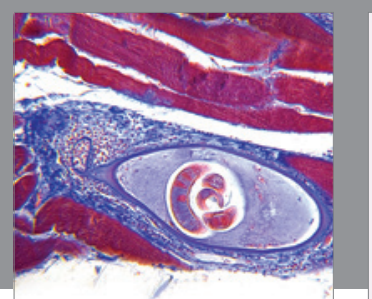

Gastroenterology Research and Practice

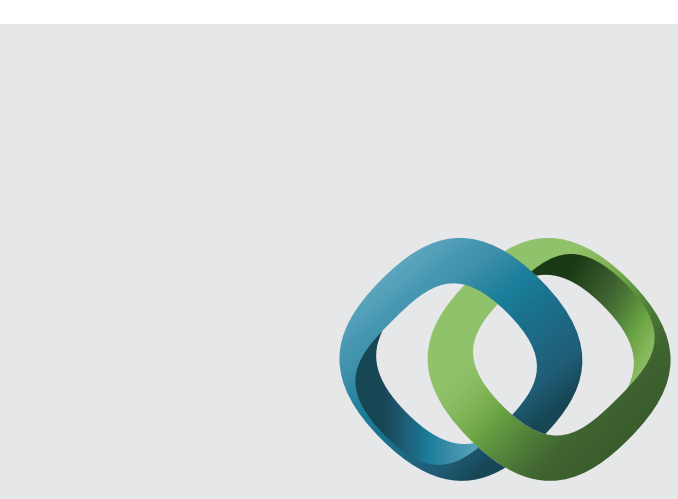

\section{Hindawi}

Submit your manuscripts at

http://www.hindawi.com
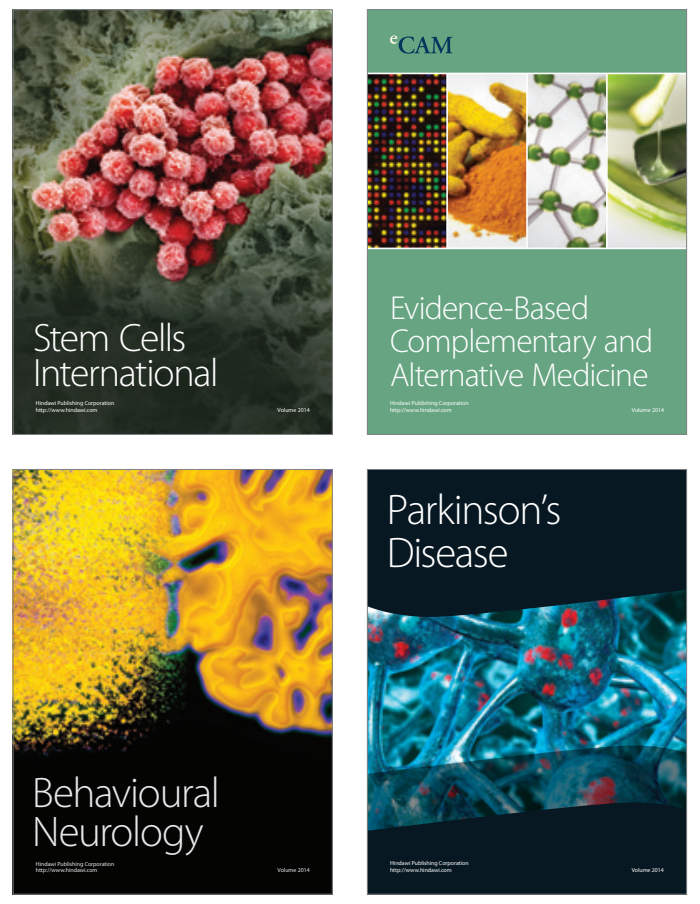
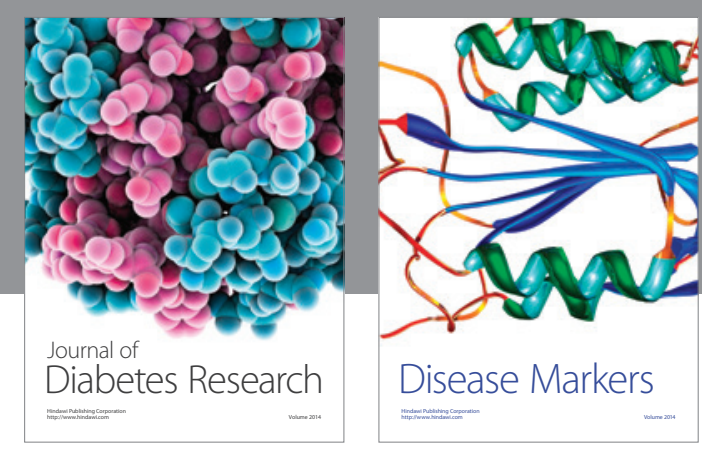

Disease Markers
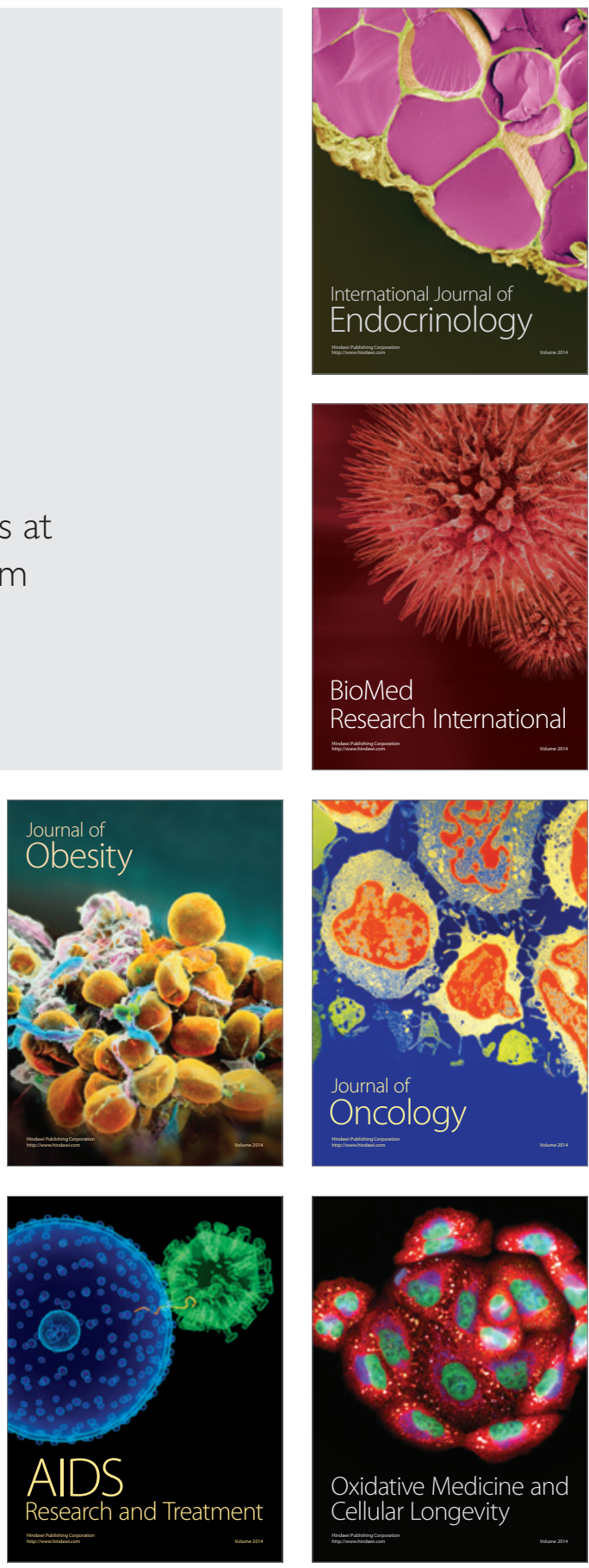